ARTICLE

https://doi.org/10.1038/s41467-019-13525-3

OPEN

\title{
Biogenesis and functions of aminocarboxypropyluridine in tRNA
}

\author{
Mayuko Takakura (1) ${ }^{1,2}$, Kensuke Ishiguro (1) ${ }^{1,2}$, Shinichiro Akichika (1D ${ }^{1}$, Kenjyo Miyauchi (i) ${ }^{1} \&$ \\ Tsutomu Suzuki (iD ${ }^{1 \star}$
}

Transfer (t)RNAs contain a wide variety of post-transcriptional modifications, which play critical roles in tRNA stability and functions. 3-(3-amino-3-carboxypropyl)uridine (acp ${ }^{3} U$ ) is a highly conserved modification found in variable- and D-loops of tRNAs. Biogenesis and functions of $\operatorname{acp}^{3} U$ have not been extensively investigated. Using a reverse-genetic approach supported by comparative genomics, we find here that the Escherichia coli yfiP gene, which we rename tapT (tRNA aminocarboxypropyltransferase), is responsible for $\operatorname{acp}^{3} \mathrm{U}$ formation in tRNA. Recombinant TapT synthesizes $\operatorname{acp}^{3} \mathrm{U}$ at position 47 of tRNAs in the presence of $S$ adenosylmethionine. Biochemical experiments reveal that acp ${ }^{3} \cup 47$ confers thermal stability on tRNA. Curiously, the $\triangle t a p T$ strain exhibits genome instability under continuous heat stress. We also find that the human homologs of tapT, DTWD1 and DTWD2, are responsible for $\operatorname{acp}^{3} \mathrm{U}$ formation at positions 20 and 20a of tRNAs, respectively. Double knockout cells of DTWD1 and DTWD2 exhibit growth retardation, indicating that $\mathrm{acp}^{3} \mathrm{U}$ is physiologically important in mammals.

\footnotetext{
${ }^{1}$ Department of Chemistry and Biotechnology, Graduate School of Engineering, The University of Tokyo, 7-3-1 Hongo, Bunkyo-ku, Tokyo 113-8656, Japan.

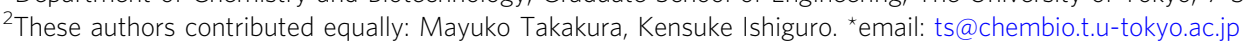


$\mathrm{T}$ he emerging field of epitranscriptomics has revealed the chemical diversity and functional importance of RNA modifications. To date, about 150 species of RNA modifications have been identified in RNA molecules from all domains of life ${ }^{1}$. Transfer (t)RNAs are especially heavily modified; indeed, more than $80 \%$ of RNA modifications found so far were discovered in tRNA molecules from various organisms. By stabilizing tRNA tertiary structure and fine-tuning decoding capability, these modifications ensure that tRNAs function properly $^{2-4}$.

A wide variety of tRNA modifications are found in the anticodon loop, especially at positions 34 and 37 . These modifications stabilize and modulate codon-anticodon interactions on the ribosome, thereby ensuring accurate and efficient decoding during translation ${ }^{2}$. The second class of modifications is clustered in the tRNA core structure formed by the D-loop, TYC-loop (Tloop), and variable-loop (V-loop). These tRNA modifications are structural modulators that contribute to correct folding and stabilization of tRNA ${ }^{3}$. Some of these modifications are also required for tRNA flexibility ${ }^{5}$. The $2^{\prime}-O$-methyl modifications found in the $\mathrm{D}$ - and T-loops, in particular, $2^{\prime}$-O-methylguanosine $(\mathrm{Gm})$ at position 18, confer conformational rigidity on the tRNA core region by fixing $\mathrm{C}^{\prime}$-endo ribose puckering ${ }^{3,6}$. Gm18 stabilizes the D-loop/T-loop interaction through base pairing with pseudouridine $(\Psi)$ at position 55 in the T-loop $7,8 . \Psi 55$ stabilizes T-loop structure with additional hydrogen bond to the phosphate-ribose backbone ${ }^{9} .5$-methyluridine $\left(\mathrm{m}^{5} \mathrm{U}\right.$, also known as ribothymidine) at position 54 in the T-loop confers thermal stability to tRNA ${ }^{10}$. In thermophilic organisms, additional modifications, including 5methyl-2-thiouridine $\left(\mathrm{m}^{5} \mathrm{~s}^{2} \mathrm{U}\right.$ or s $\left.\mathrm{s}^{2} \mathrm{~T}\right)$ and archaeosine $(\mathrm{G}+)$, are present in the tRNA core region ${ }^{11-13}$. These modifications stabilize tRNAs, enabling cell growth at high temperature ${ }^{10,14,15}$. The modifications in the core region play crucial roles in determining not only the physicochemical properties of tRNAs but also their cellular stability. Because properly modified mature tRNAs are required for accurate and efficient translation, living organisms have evolved a control quality system that degrades hypomodified tRNAs ${ }^{16,17}$.

3-(3-amino-3-carboxypropyl)uridine $\left(\operatorname{acp}^{3} \mathrm{U}\right)$ is a widely conserved modification found in tRNA core region in bacteria and eukaryotes ${ }^{1,18,19}$ (Fig. 1a). The 3-amino-3-carboxypropyl (acp) group is attached to the $\mathrm{N} 3$ atom of the uracil base to prevent it from engaging in Watson-Crick base pairing. In Escherichia coli, acp $^{3} \mathrm{U}$ is present at position 47 in the V-loop of tRNAs for Arg2, Ile1, Ile2, Ile2v, Lys, Met, Phe, Val2A, and Val2B (Fig. 1a) ${ }^{20}$. In eukaryotes, including human, rat and Drosophila, acp ${ }^{3} \mathrm{U}$ occurs at positions 20 and 20a in the D-loop of several cytoplasmic tRNAs (Fig. 1a) ${ }^{20}$. In Trypanosoma brucei, acp ${ }^{3} \mathrm{U}$ and its dihydrouridine derivative $\left(\mathrm{acp}^{3} \mathrm{D}\right)$ are present at positions 20 and 47 , respectively,

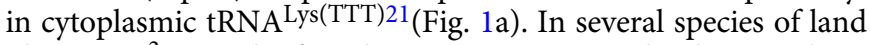
plants, $\mathrm{acp}^{3} \mathrm{U}$ can be found at positions $20 \mathrm{a}$ and $20 \mathrm{~b}$ in nuclearencoded tRNAs, and at position 47 in tRNAs encoded in plastid and mitochondrial DNAs ${ }^{20}$.

An $\operatorname{acp}^{3} \mathrm{U}$ derivative, 1-methyl-3-(3-amino-3-carboxypropyl) pseudouridine $\left(\mathrm{m}^{1} \mathrm{acp} \mathrm{p}^{3} \Psi\right)$, is present at the P-site of small subunit of the eukaryotic and archaeal ribosome: positions 1191 and 1248 in $18 \mathrm{~S}$ rRNA from S. cerevisiae and human, respectively ${ }^{22-24}$. In human, $\mathrm{m}^{1} \mathrm{acp}^{3} \Psi 1248$ is synthesized via three steps. The biogenesis is initiated by pseudouridylation mediated by H/ACA snoRNP bearing SNORA $13^{25}$, followed by methylation catalyzed by EMG1 ${ }^{26}$. Finally, TSR3 transfers the acp group of $S$-adenosylmethionine $(\mathrm{SAM})$ to form $\mathrm{m}^{1} \mathrm{acp}^{3} \Psi^{27,28}$. TSR3 contains a domain similar to SPOUT-class RNA methyltransferase ${ }^{28}$. The crystal structure of the archaeal homolog of TSR3 in complex with SAM revealed the molecular basis of $\operatorname{acp}^{3} \mathrm{U}$ formation ${ }^{28}$, although the enzymatic activity of TSR3 has never been described.
Reduction in the level of the acp modification causes accumulation of $18 \mathrm{~S}$ rRNA precursors and a reduction in the level of $40 \mathrm{~S}$ ribosomal subunit, indicating that this modification is involved in 40 S subunit assembly 28,29 .

Despite the high conservation of the $\operatorname{acp}^{3} \mathrm{U}$ modification in tRNAs from bacteria and eukaryotes, the biogenesis and physiological roles of this modification remain to be determined. The solution structure of the $\mathrm{acp}^{3} \mathrm{U}$ nucleoside does not affect its sugar conformation ${ }^{30}$. Structural analysis of D-acp ${ }^{3} \mathrm{U}-\mathrm{A}$ trinucleotide suggested that $\mathrm{acp}^{3} \mathrm{U} 20 \mathrm{a}$ binds to $\mathrm{Mg}^{2+}$, and this interaction may stabilize the local conformation of tRNA ${ }^{31}$. Chemical acetylation of the amino group of $\mathrm{acp}^{3} \mathrm{U} 47$ in E. coli tRNA $\mathrm{Ph}^{\text {Phe }}$ does not affect its binding affinity for phenylalanyl-tRNA synthetase or the ribosome $\mathrm{e}^{32}$. In the tertiary structure of tRNAs $\mathrm{s}^{33-35}$, the acp group of acp ${ }^{3} \mathrm{U} 47$ is oriented toward the solvent side of the tRNA structure and does not directly interact with the other residues. In 1974, enzymatic formation of $\operatorname{acp}^{3} \mathrm{U}$ in tRNA was carried out using E. coli lysate ${ }^{36}$. Specifically, Nishimura's group successfully reconstituted $a^{3} \mathrm{CP}^{3} \mathrm{H}$ formation in tRNA ${ }^{\text {Phe }}$ in the presence of SAM, and demonstrated that the acp group of SAM is transferred to tRNA to form acp ${ }^{3} \mathrm{U} 47$ (Fig. 1b).

To achieve a deeper understanding of $\operatorname{acp}^{3} \mathrm{U}$ modification in tRNAs, it is necessary to identify the enzyme responsible for generating it. For systematic search for genes responsible for RNA modifications, we developed a genetic screening method called ribonucleome analysis, which takes advantage of mass spectrometric analysis of RNA modification ${ }^{37}$. We use liquid chromatography/mass spectrometry (LC/MS) to systematically analyze total nucleosides of RNAs obtained from a series of strains harboring knockouts in uncharacterized genes. If a target RNA modification is absent in a certain knockout strain, we can identify the gene dedicated to biogenesis of the modification in a reverse-genetic manner. We have discovered dozens of genes responsible for RNA modifications in tRNAs ${ }^{38-41}$, rRNAs ${ }^{42,43}$, and $m R N A s^{44}$. In this study, we apply ribonucleome analysis assisted by comparative genomics to identify an $E$. coli gene $y f i P$ (renamed as tapT) responsible for $\mathrm{acp}^{3} \mathrm{U} 47$ formation. We successfully reconstitute formation of acp ${ }^{3} \mathrm{U} 47$ by recombinant TapT protein in the presence of SAM. Measurements of melting temperature reveal that acp ${ }^{3} \mathrm{U} 47$ stabilizes tRNA by $3^{\circ} \mathrm{C}$. We observe genome instability of the $\triangle t a p T$ strain under continuous heat stress, indicating that this modification plays a physiological role in bacteria. We also identify eukaryotic homologs of tapT, including the human homologs DTWD1 and DTWD2, and show that they are responsible for the formation of $\operatorname{acp}^{3} \mathrm{U}$ at positions 20 and 20a of tRNAs, respectively. In human cells, double knockout of DTWD1 and DTWD2 causes slow growth, indicating that $\mathrm{acp}^{3} \mathrm{U}$ is also physiologically important in mammals.

\section{Results}

E. coli $y f i P$ is responsible for acp ${ }^{3} \mathrm{U} 47$ formation on tRNAs. To identify the gene responsible for $\mathrm{acp}^{3} \mathrm{U} 47$ formation in E. coli, we used comparative genomics to narrow down the candidate genes (Fig. 2a). Because little information was available about posttranscriptional modifications in the other bacterial tRNAs, we conducted nucleoside analyses of total RNAs from several bacterial species, Acidimicrobium ferrooxidans, Synechocystis sp. PCC 6803, Thermus thermophilus HB27, Bacillus subtilis str. 168, and Mycoplasma mobile $163 \mathrm{~K}$, and found that none of them contained acp $^{3} \mathrm{U}$ (Supplementary Fig. 1). By contrast, in protists, $\operatorname{acp}^{3} \mathrm{U}$ is present in T. brucei cytoplasmic tRNA $\left.\mathrm{Lys}^{\mathrm{LuUU}}\right) 21$, and we confirmed the presence of $\mathrm{acp}^{3} \mathrm{U}$ in the same tRNA from Leishmania major ${ }^{45}$. Using these data, we performed a comparative genomics analysis using the Microbial Genome Database $^{46}$. Among 4144 E. coli ORFs, we selected 65 genes commonly 


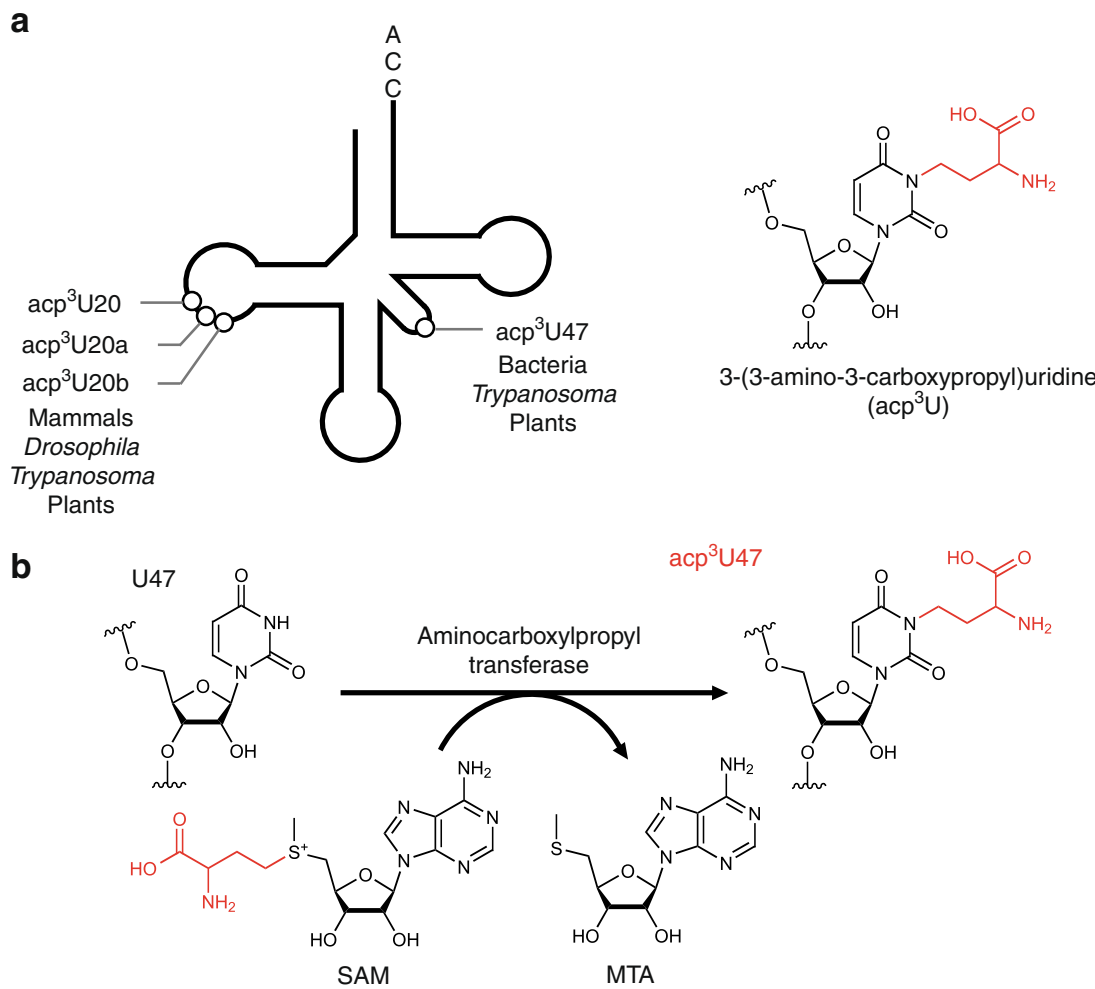

Fig. 1 acp $^{3} \mathbf{U}$ is a prevalent tRNA modification widely conserved in eukaryotes and bacteria. a Positions of acp ${ }^{3} U$ modification in tRNA (left panel). Chemical structure of 3-(3-amino-3-carboxypropyl)uridine $\left(\operatorname{acp}^{3} \mathrm{U}\right.$ ) (right panel). The acp group is colored in red. acp ${ }^{3} \mathrm{U}$ is present at position 20 in mammals, Drosophila and Trypanosoma, at position 20a in mammals, Drosophila and plants, and at position 20b in plants. b Biosynthetic mechanism of $\operatorname{acp}^{3} \mathrm{U}$ modifications in tRNA and rRNA. The acp group of S-adenosylmethionine (SAM) is transferred to generate acp ${ }^{3} \mathrm{U}$ modification in RNA with release of S-methyl-5'-thioadenosine (MTA).

present in E. coli, as well as in the two protists (T. brucei and L. major), but absent in the five bacteria mentioned above (Fig. 2a, b). Based on the annotation of each gene in the EcoCyc database ${ }^{47}$, we then narrowed down the list of candidates to six genes with unknown functions. Finally, we chose $y f i P$ as a strong candidate for $\mathrm{acp}^{3} \mathrm{U}$ formation (Fig. $2 \mathrm{~b}$ ). $y f i P$ belongs to unknown orthologous group COG3148 that contains an uncharacterized DTW domain. According to computational analyses, YfiP has a SPOUT-like methyltransferase structure ${ }^{48}$. Moreover, COG3148 was referred to the DTWD2 family (KOG4382), and classified into the TDD superfamily, which includes the TSR3 (COG2042), DTWD1 (KOG3795), and DTWD2 families ${ }^{49}$.

To examine whether $y f i P$ is responsible for $\mathrm{acp}^{3} \mathrm{U} 47$ formation in tRNAs, we performed LC/MS nucleoside analysis of total RNAs from E. coli WT and $\Delta y f i P$ strains (Fig. 2c). As expected, no acp $^{3} U$ was detected in the $\Delta y f i P$ strain, but acp ${ }^{3} U$ was restored by ectopic expression of a plasmid-encoded $y f i P$ gene. These results clearly demonstrated that $y f i P$ is essential for $\operatorname{acp}^{3} \mathrm{U}$ formation in E. coli. Hereafter, we refer to yfiP as tapt (tRNA aminocarboxypropyltransferase).

TapT catalyzes acp ${ }^{3} \mathrm{U} 47$ formation. Next, we performed in vitro reconstitution of $\mathrm{acp}^{3} \mathrm{U} 47$ using the recombinant Tap $\mathrm{T}$ protein. As a substrate, we isolated $E$. coli tRNA $^{\text {Met }}$ lacking $\operatorname{acp}^{3} \mathrm{U}$ from the $\Delta t a p T$ strain. After the reaction, the tRNA was digested by RNase $\mathrm{T}_{1}$ and analyzed by LC/MS. We clearly detected an RNA fragment containing acp ${ }^{3} \mathrm{U}$ in the presence of both TapT and SAM (Fig. 2d). TapT efficiently introduced acp ${ }^{3} \mathrm{U} 47$ on unmodified $\mathrm{tRNA}^{\text {Met }}$ transcribed in vitro (Supplementary Fig. 2), suggesting that the other tRNA modifications are not required for $\operatorname{acp}^{3} U$ formation. The fragment was further probed by collision- induced dissociation, confirming that $\operatorname{acp}^{3} \mathrm{U}$ was introduced at position 47 (Fig. 2e). These data clearly demonstrated that TapT is a tRNA-modifying enzyme that catalyzes the SAM-dependent aminocarboxypropyl transfer reaction to form acp ${ }^{3} \mathrm{U} 47$.

$\operatorname{acp}^{3} U$ confers thermal stability on tRNA. Given that $\operatorname{acp}^{3} U$ is a modification in the tRNA core region, we investigated whether $\operatorname{acp}^{3} \mathrm{U} 47$ stabilizes the tertiary structure of tRNA. We prepared $E$. coli tRNA $^{\text {Met }}$ transcripts with or without acp ${ }^{3} \mathrm{U} 47$ and compared their melting profiles. As temperature increased, the tRNA gradually melted, and its hyperchromicity increased (Fig. 2f). The unmodified tRNA started melting around at $40^{\circ} \mathrm{C}$, whereas the tRNA containing acp ${ }^{3} \mathrm{U} 47$ remained stable even at $50^{\circ} \mathrm{C}$. The hyperchromicity of the modified tRNA was lower than that of the unmodified one throughout the course of the heating process. The melting temperature $\left(T_{\mathrm{m}}\right)$ values of the tRNA with and without acp ${ }^{3} \mathrm{U} 47$ were 70.0 and $66.7^{\circ} \mathrm{C}$, respectively (Fig. 2f). Thus, a single $\operatorname{acp}^{3} \mathrm{U}$ modification thermally stabilized this tRNA by $3{ }^{\circ} \mathrm{C}$.

Motility defect in $E$. coli $\Delta$ tapT strain. To investigate the physiological roles of acp ${ }^{3} \mathrm{U} 47$ in $E$. coli, we explored the phenotypic features of the $\Delta t a p T$ strain. Because acp ${ }^{3} U 47$ thermally stabilizes tRNA, we compared the cell growth of WT and $\Delta t a p T$ strains cultured at different temperatures (Supplementary Fig. 3a), but observed no significant difference in cell growth even at $42^{\circ} \mathrm{C}$. However, we happened to find a morphological phenotype unique to the $\Delta t a p T$ strain when cultured continuously at high temperature. After 3 days cultivation at $42{ }^{\circ} \mathrm{C}$, small colonies suddenly appeared in the $\Delta \operatorname{tap} T$ strain (Fig. $3 \mathrm{a}, \mathrm{b}$ ), although no such colonies were detected in the first 2 days. Small colonies 
a

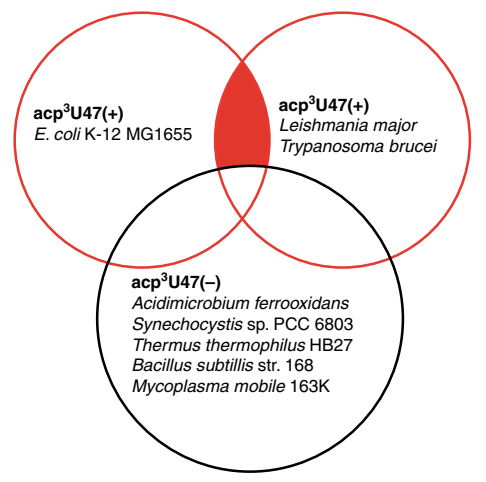

c

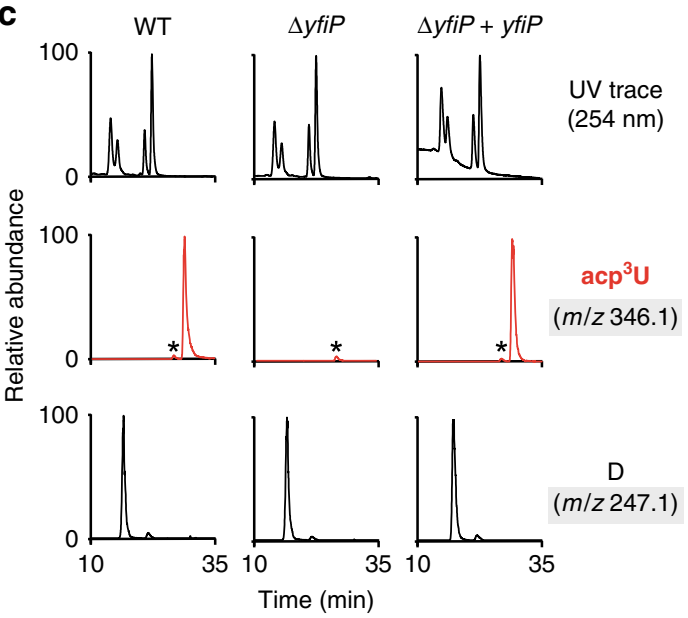

b

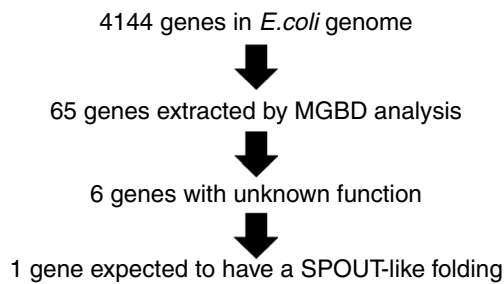

d

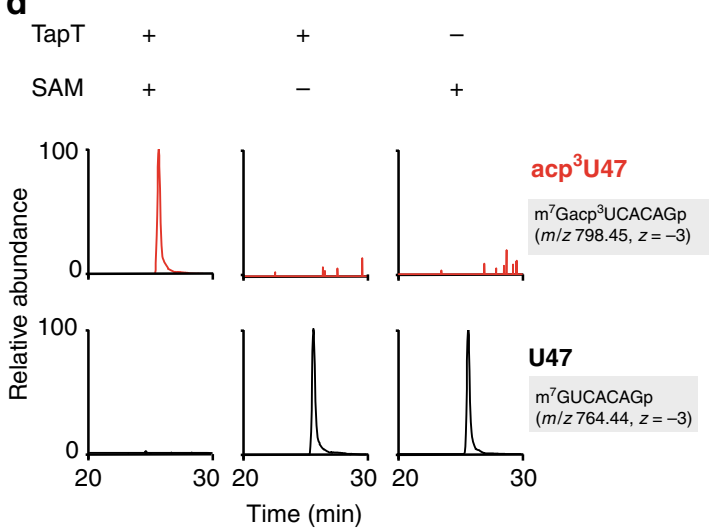

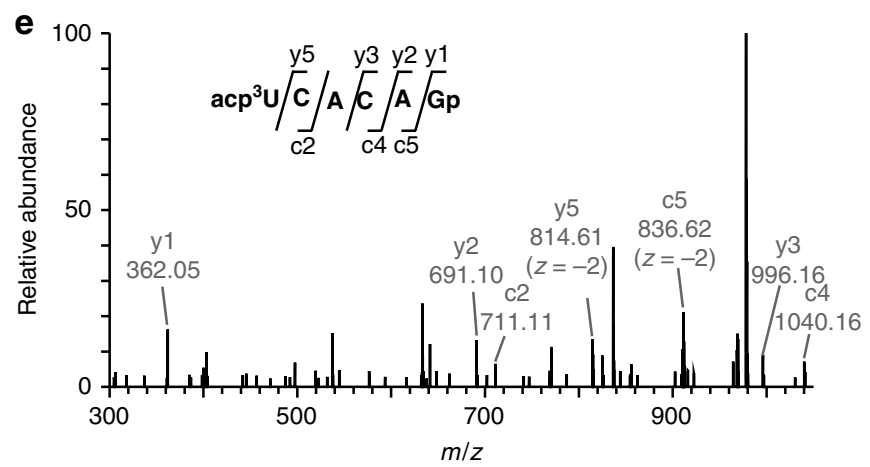

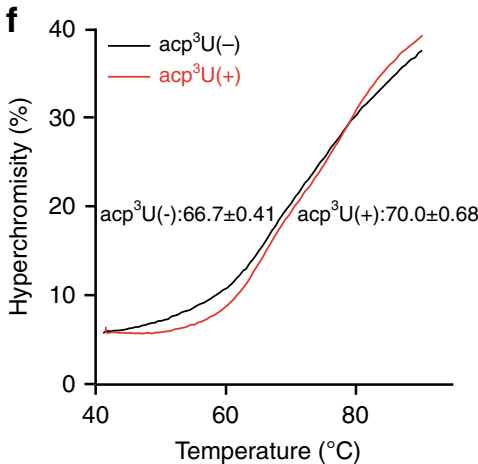

Fig. 2 yfiP is responsible for acp ${ }^{3} \mathbf{U} 47$ formation in E. coli. a Venn diagram to narrow down the candidates by comparative genomics. Each circle represents the gene sets that are commonly present in the organisms with $(+)$ or without $(-)$ acp ${ }^{3} U$. Red circles represent groups of organisms that have $\operatorname{acp}^{3} \cup 47$, whereas the black circle represents organisms that do not have acp ${ }^{3} 44$. Candidate genes lie in the red-filled region. $\mathbf{b}$ The process of narrowing down the candidate genes responsible for acp ${ }^{3} U 47$ formation by comparative genomics. c Mass spectrometric nucleoside analyses of tRNA modifications from E. coli WT (left panels), $\Delta y f i P$ (center panels), an $\Delta y$ fiP rescued by plasmid-encoded yfiP (right panels). UV chromatogram (top) and extracted ion chromatograms (XICs) of the proton adducts of nucleosides for $\operatorname{acp}^{3} \mathrm{U}$ (middle) and $\mathrm{D}$ (bottom). Asterisks indicate nonspecific peaks with the same $m / z$ value. $\mathbf{d}$ In vitro reconstitution of $\mathrm{acp}^{3} \mathrm{U} 47$ in $E$. coli tRNA Met by recombinant TapT in the presence or absence of SAM. XICs show the indicated negative ions of the RNase $T_{1}$-digested fragments with (top) or without (bottom) acp ${ }^{3} U 47$. e Collision-induced dissociation spectrum of the acp ${ }^{3} U$-containing fragment of the tRNAMet transcript, in which acp ${ }^{3} U$ was reconstituted in vitro. The precursor ion is $\mathrm{m} / z 678.43$. The product ions were assigned on the corresponding sequence as described ${ }^{93}$. The data confirmed the presence of $\operatorname{acp}^{3} \mathrm{U}$ at position 47. $\mathbf{f}$ Melting curves of the in vitro transcribed tRNAMet with (red lines) or without (black lines) acp ${ }^{3} \cup 47$ modification. $T_{m}$ values were determined based on the inflection point of the melting curve. Data represent average values of triplicates, with s.d. $P<4.1 \times 10^{-3}$ by Student's $t$ test. Source data are provided as a Source Data file.

were not detected in the WT strain, in the $\Delta$ tapT strain cultured at $37^{\circ} \mathrm{C}$, or in the $\Delta t a p T$ strain complemented with plasmidencoded tapT gene (Fig. 3c).

We isolated and characterized small and normal-sized $\Delta t a p T$ colonies cultured for 4 days at $42{ }^{\circ} \mathrm{C}$, and then examined the growth and motility of these cells. We observed no significant difference in cell growth between small and normal colonies (Supplementary Fig. 3b). Cell motility was examined based on swimming activity on soft agar plates. As shown in Fig. 3d, cells derived from normal colonies swam well, and the diameter of the halo reached $2.90 \pm 0.21 \mathrm{~cm}$, whereas cells derived from small colonies did not move and instead remained near the original spot $(0.55 \pm 0.04 \mathrm{~cm})$, suggesting that the small colony phenotype of the $\Delta t a p T$ strain originates from a motility defect. When we sought to reverse the phenotype by introducing plasmid-encoded tapT into cells derived from small colonies, we observed no 

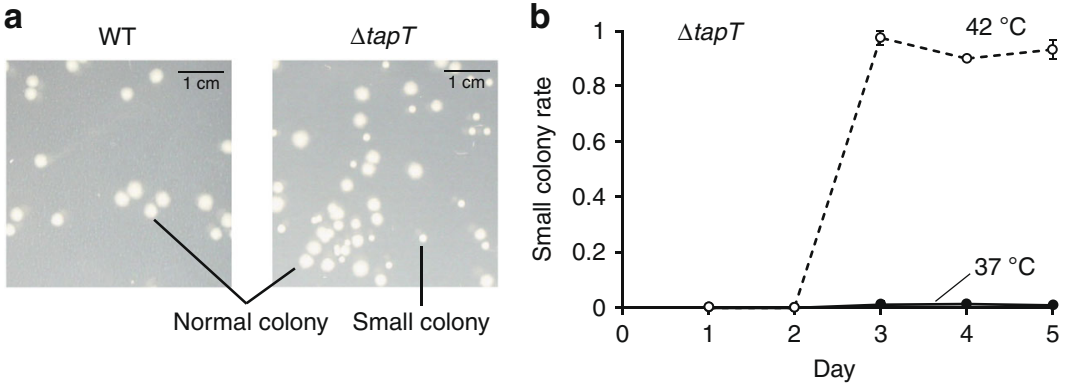

d

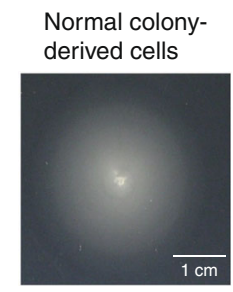

$2.90 \pm 0.21 \mathrm{~cm}$

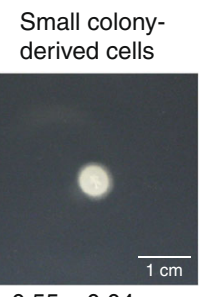

$0.55 \pm 0.04 \mathrm{~cm}$
Small colonyderived cells $+\mathrm{p}-\operatorname{tap} T$

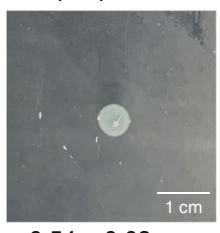

$0.54 \pm 0.08 \mathrm{~cm}$ e Small colony $37^{\circ} \mathrm{C}$

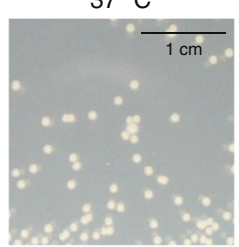

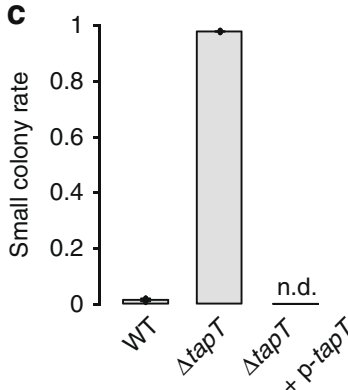

Small colony $+\mathrm{p}$-tap $T$ $37^{\circ} \mathrm{C}$

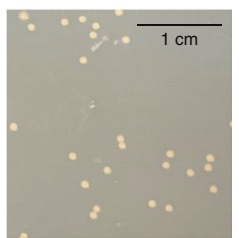

Fig. 3 Lack of acp $\mathbf{3}^{\mathbf{U}} \mathbf{4 7}$ results in genome instability in E. coli. a Morphological alteration of WT (left) or $\Delta$ tapT (right) strains after cultivation at $42{ }^{\circ} \mathrm{C}$ for 4 days. Small colonies appeared in the $\Delta \operatorname{tap} T$ strain. b Frequencies of small colony appearance of $\Delta$ tap $T$ strain with continuous cultivation at $37^{\circ} \mathrm{C}$ (closed circles) and $42^{\circ} \mathrm{C}$ (open circles). Data represent average values of biological triplicates, with s.d. Source data are provided as a Source Data file.

c Frequencies of small colonies in WT, $\Delta \operatorname{tap} T$, and $\Delta \operatorname{tap} T$ overexpressing tap $T$ after cultivation at $42{ }^{\circ} \mathrm{C}$ for 4 days. Data represent average values of biological triplicates, with s.d. Source data are provided as a Source Data file. d Motility test of the normal colony-derived cells (left), small colony-derived cells (middle), and small colony-derived cells overexpressing tapT (right). Diameter of halo was defined as the length of the line segment which passes through the center and the ends of the halo. Data represent average values of biological replicates $(n=5)$, with s.d. Source data are provided as a Source Data file. e Morphology of the small colony-derived cells (left) and the small colony-derived cells overexpressing tapT (right). Overnight saturated cultures were diluted, spotted onto LB plates, and cultivated at $37^{\circ} \mathrm{C}$.

normal colonies on the plate (Fig. 3e). Supporting this result, lack of motility on soft agar was not restored by tapT complementation $(0.54 \pm 0.08 \mathrm{~cm})$ (Fig. $3 \mathrm{~d})$. We confirmed restoration of $\mathrm{acp}^{3} \mathrm{U}$ in the small colony-derived $\Delta \operatorname{tap} T$ strain by introduction of plasmid-encoded tapT gene (Supplementary Fig. 3c). Thus, the phenotype is caused by genomic mutations.

tRNA modification-deficient strains exhibit a moderate mutator phenotype mediated by the translational stress-induced mutagenesis (TSM) pathway ${ }^{50,51}$. Lack of $N^{6}$-isopentenyladenosine $\left(i^{6} \mathrm{~A}\right)$ in tRNAs in the $\triangle m i a A$ strain induces transversiontype DNA mutation ${ }^{50}$. If similar translation stress is induced by loss of $\operatorname{acp}^{3} \mathrm{U}$ in the $\Delta t a p T$ strain, the genomic mutation linked to the small colony phenotype might be enhanced by the TSM pathway $^{51}$. To measure the genomic mutation rate of the $\Delta t a p T$ strain, we performed a mutator assay ${ }^{52}$. Specifically, we cultured the cells continuously at $42{ }^{\circ} \mathrm{C}$ and then examined their resistance to nalidixic acid (Supplementary Fig. 4). As a positive control, we used the $\Delta m u t S$ strain, in which the DNA mismatch repair system is inactive; 53 a number of small colonies arose on the plate, whereas the negative control strain $(\triangle y a g A)$ showed a lower mutation rate. As reported, the $\triangle m i a A$ strain exhibited a mild mutator phenotype. However, in contrast to our speculation, the mutation rate of the $\Delta t a p T$ strain was in the same range as that of the control strain, suggesting that the small colony phenotype of the $\Delta \operatorname{tap} T$ strain under heat stress cannot be explained by a mutator phenotype.

DTWD1 and DTWD2 mediate $\operatorname{acp}^{3} U$ formation in human tRNAs. In mammals, acp ${ }^{3} \mathrm{U}$ is present at position 20 of tRNA ${ }^{\text {Tyr }} 54$ and position 20 a of tRNA ${ }^{\text {Asn }} 55$. We reanalyzed high throughput sequencing of human tRNAs ${ }^{56}$ and observed an apparent U-to-C conversion at positions 20 and $20 \mathrm{a}$ of these tRNAs, indicating that $\operatorname{acp}^{3} \mathrm{U}$ causes misincorporation during cDNA synthesis. Based on the misincorporation signatures at positions 20 and 20a of several human tRNAs, we predicted the presence of acp ${ }^{3} \mathrm{U} 20$ in two tRNAs for Cys(GCA) and Tyr, and $\mathrm{acp}^{3} \mathrm{U} 20 \mathrm{a}$ in three tRNAs for Asn(GTT), Ile(AAT), and Ile(TAT). We then isolated these cytoplasmic tRNAs from HEK293T cells and analyzed the tRNA modifications by capillary LC-nanoelectrospray ionization (ESI)-MS (RNA-MS) ${ }^{37}$. We confirmed the presence of $\operatorname{acp}^{3} \mathrm{U}$ at positions 20 and 20a in these five tRNA species (Fig. $4 a$, b and Supplementary Fig. $5 \mathrm{a}-\mathrm{c}$ ). In addition, we found $\mathrm{acp}^{3} \mathrm{D}$ at position 20a of tRNAlle(AAT) (Fig. 4b).

As described above, tapT belongs to the DTWD2 family and TDD superfamily ${ }^{49}$. Two paralogs of DTW-containing proteins in the TDD superfamily, DTWD1 and DTWD2, are encoded in the human genome, and we hypothesized that these proteins are responsible for $\operatorname{acp}^{3} U$ at positions 20 and 20a. To examine this speculation, we knocked out each of these proteins in HEK293T cells using the CRISPR-Cas9 system. Two isoforms of human DTWD2 with different first exons are produced by alternative splicing: DTWD2L (long) and DTWD2S (short). (Supplementary Fig. 6a). To delete both isoforms, we designed sgRNAs targeting exons 2 and 3 (Supplementary Fig. 6a). We also constructed DTWD2L-specific KO cell line by targeting the exon 1 of DTWD2L. Finally, we constructed DTWD1/DTWD2 double$\mathrm{KO}$ cell lines. Genotyping of each $\mathrm{KO}$ cell line confirmed frameshift mutations in both alleles (Supplementary Fig. 6b).

We then isolated five individual tRNAs bearing $\operatorname{acp}^{3} U$ from a series of $\mathrm{KO}$ strains and examined their modification status by RNA-MS. In the DTWD1 KO cells, the level of acp ${ }^{3} \mathrm{U} 20$ in two tRNAs for Cys(GCA) and Tyr were drastically reduced, with the modified bases converted to D20 and U20, although a small percentage of $\mathrm{acp}^{3} \mathrm{U} 20$ remained (Fig. $4 \mathrm{a}$ and Supplementary Fig. 5a). We observed no effect on $\operatorname{acp}^{3} \mathrm{U} 20 \mathrm{a}$ or acp ${ }^{3} \mathrm{D} 20 \mathrm{a}$ in the other three tRNAs. No $\operatorname{acp}^{3} \mathrm{U} 20$ was detected in the DTWD1/DTWD2 double-KO cells (Fig. 4a and Supplementary 

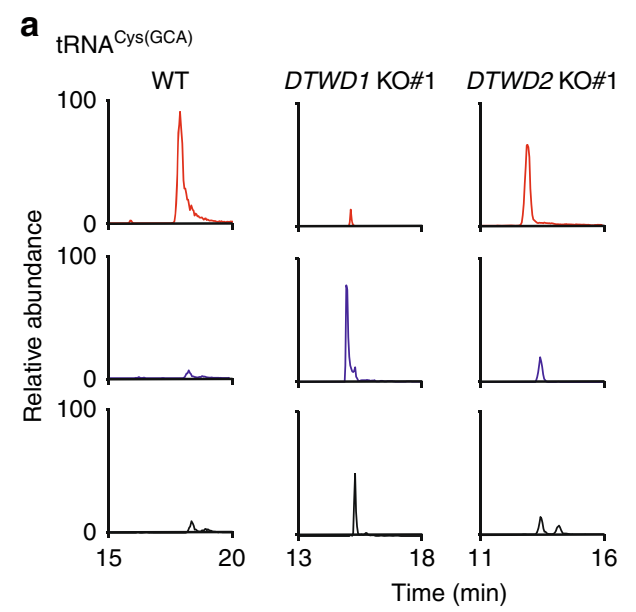

DTWD1/DTWD2

double KO\#1

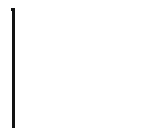

acp $^{3} U 20$

acp UCAGp

$(m / z 1098.18 z=-1)$

b tRNA $^{\| l(A A T)}$
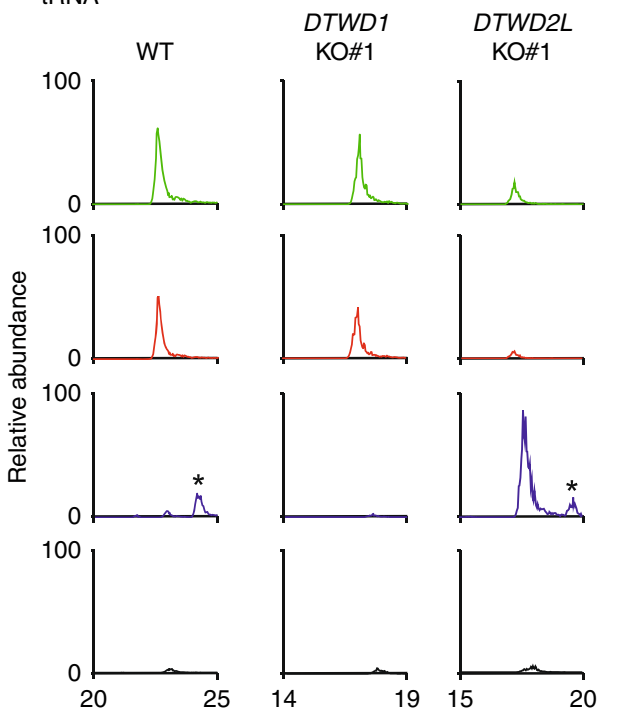

DTWD2

DTWD1/DTWD2

$\mathrm{KO \# 1}$

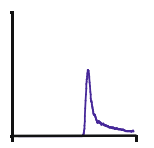

D20

DAGp

$(m / z 999.14 z=-1)$

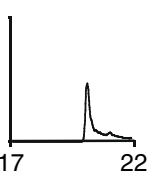

U20

UAGp

$m / z 997.13 z=-1)$
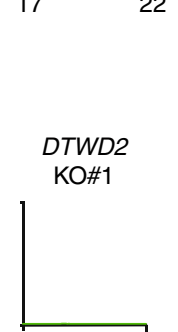

double KO\#1

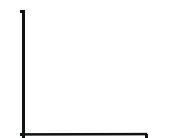

acp $^{3} \mathrm{D} 20 \mathrm{a}$

Dacp32DAGp

$(m / z 703.61, z=-2)$
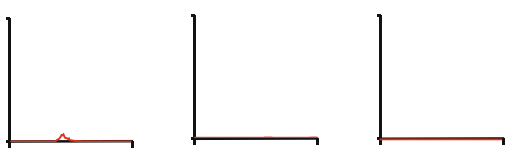

$\mathrm{acp}^{3} \mathrm{U} 20 \mathrm{a}$

Dacp ${ }^{3}$ UAGp

$(m / z 702.60, z=-2)$
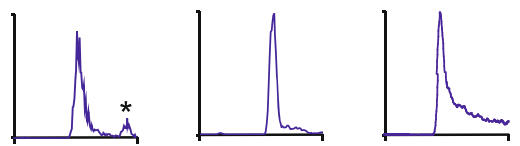

D20a

DDAGp

$(m / z 653.09, z=-2)$
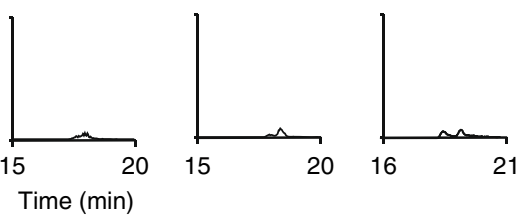

U20a

DUAGp

$(m / z 652.08, z=-2)$

C

A
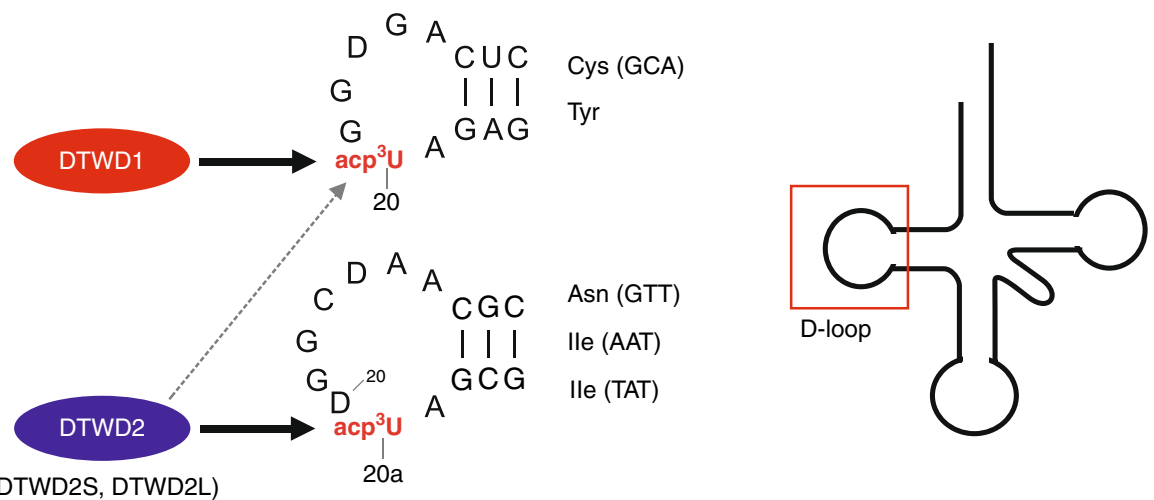

(DTWD2S, DTWD2L)

$20 a$

Fig. 4 Human DTWD1 and DTWD2 are responsible for acp $\mathbf{3}^{\mathbf{U}}$ formation. a Mass spectrometric analyses of acp ${ }^{3} \mathrm{U}_{20} \mathrm{in}$ tRNACys(GCA) isolated from WT (left), DTWD1 KO (left middle), DTWD2 KO (right middle), and DTWD1/DTWD2 double-KO (right) strains. XICs show corresponding negative ions of the RNase $T_{1}$-digested fragments as indicated on the right-hand side of each chromatogram. Mass spectra of acp ${ }^{3} \mathrm{D}$ - or $\mathrm{D}$-containing fragments overlap with the isotopic peaks of $a_{c p}{ }^{3} \mathrm{U}$ - or $\mathrm{U}$-containing fragments. The peak intensities of these fragments are normalized in consideration of those isotopes. Asterisk indicates nonspecific peaks with the same $\mathrm{m} / \mathrm{z}$ value. b Mass spectrometric analyses of acp ${ }^{3} \mathrm{U} 20 \mathrm{a}$ in tRNAlle(AAT) isolated from WT (left), DTWD1 KO (left middle), DTWD2L KO (middle), DTWD2 KO (right middle), and DTWD1/DTWD2 double-KO (right) strains. c Substrate specificity of human DTWD1 and DTWD2. DTWD1 is responsible for acp ${ }^{3} \mathrm{U} 20$ formation in tRNAs for Cys (GCA) and Tyr, whereas DTWD2 is responsible for acp 3 U20a formation in tRNAs for Asn(GTT), Ile(AAT), and Ile(TAT). DTWD2 also has a weak activity to form acp ${ }^{3}$ U20. The sequence of tRNACys and tRNAAsn are shown. 
Fig. 5a), indicating that DTWD1 is primarily responsible for introducing acp ${ }^{3} \mathrm{U} 20$, whereas DTWD2 plays a supportive role in $\mathrm{acp}^{3} \mathrm{U} 20$ formation (Fig. 4c). On the other hand, $\mathrm{acp}^{3} \mathrm{U} 20 \mathrm{a}$ and $\mathrm{acp}^{3} \mathrm{D} 20 \mathrm{a}$ were deficient, and replaced by D20a, in three tRNAs for Asn(GTT), Ile(AAT), and Ile(TAT) in the DTWD2 KO cells (Fig. 4b and Supplementary Fig. 5b, c), suggesting that DTWD2 has a strong specificity for introducing acp ${ }^{3}$ U20a and $a^{3} p^{3}$ D20a (Fig. 4c). These data demonstrated that DTWD1 and DTWD2 have different substrate preferences for tRNAs, and are responsible for the biogenesis of $\mathrm{acp}^{3} \mathrm{U} 20$ and $\mathrm{acp}^{3} \mathrm{U} 20 \mathrm{a}$, respectively.

Regarding two isoforms of DTWD2, we isolated tRNA ${ }^{\text {Ile(AAT) }}$ from DTWD2L KO cells, and analyzed the status of acp ${ }^{3} \mathrm{U} 20 \mathrm{a}$ modification (Fig. 4b). Both $\mathrm{acp}^{3} \mathrm{U} 20 \mathrm{a}$ and $\mathrm{acp}^{3} \mathrm{D} 20 \mathrm{a}$ partially decreased, and instead D20a increased. These data clearly demonstrated that DTWD2L actually has an activity for acp ${ }^{3} \mathrm{U}$ (D)20a formation, and DTWD2S is redundantly responsible for the remaining modification.

Intriguingly, D20 or D20a appeared upon knockout of DTWD1 or DTWD2 (Fig. 4a, b and Supplementary Fig. 5a-c). D20 and D20a are present in other tRNA species that do not contain $\operatorname{acp}^{3} \mathrm{U}$ and have been proposed to be introduced by DUS2 and DUS4L, respectively ${ }^{57,58}$. Thus, DTWD1/2-mediated $\operatorname{acp}^{3} \mathrm{U}$ formation inhibits D20(a) formation.

Growth reduction of $D T W D 1$ and $D T W D 2$ double knockout cells. We next investigated the physiological significance of $\operatorname{acp}^{3} \mathrm{U}$ modifications in human tRNAs. In comparison with WT HEK293T cells, little growth reduction was observed in DTWD1 and DTWD2 single-KO cells (Fig. 5a), whereas the DTWD1/DTWD2 double-KO cells exhibited a severe growth phenotype (Fig. 5a), indicating that DTWD1 and DTWD2 play redundant roles in supporting normal cell growth. Given that tRNA modifications are required for the cellular stability of tRNAs ${ }^{16}$, we asked whether loss of $\operatorname{acp}^{3} \mathrm{U}$ modification affects the steady-state level of tRNAs. To this end, we performed Northern blotting to compare the steady-state levels of four tRNAs between WT HEK293T and DTWD1/DTWD2 double-KO cells (Supplementary Fig. 7a, b). No significant difference was observed for any tRNAs, suggesting that loss of $a_{c p}^{3} U$ does not affect tRNA stability.

Finally, we analyzed the subcellular localization of DTWD1 and DTWD2. For this purpose, FLAG-tagged DTWD1, DTWD2L, and DTWD2S were transiently expressed in HeLa cells and detected by immunostaining (Fig. 5b). DTWD1 was predominantly localized in nucleus, suggesting that DTWD1mediated acp ${ }^{3} \mathrm{U} 20$ formation takes place in nucleus. By contrast, the two isoforms of DTWD2 exhibited different subcellular localization: DTWD2L was cytoplasm, whereas DTWD2S was widely diffused throughout the cells but concentrated in the nucleus. Thus, acp ${ }^{3} \mathrm{U} 20 \mathrm{a}$ formation occurs in both the cytoplasm and the nucleus.

\section{Discussion}

In this study, we discovered that the tap $T$ gene is responsible for acp $^{3} \mathrm{U} 47$ formation in E. coli tRNAs. TapT was the last unknown tRNA-modifying enzyme in E. coli. We successfully reconstituted in vitro $\mathrm{acp}^{3} \mathrm{U} 47$ formation with the recombinant TapT protein in the presence of SAM, revealing that TapT catalyzes acp transfer from SAM to tRNA. The acp moiety of SAM is also utilized for the synthesis of various biomolecules ${ }^{59}$, including bacterial betaine lipid, nocardicin, microcin $\mathrm{C}\left(\mathrm{McC}^{1177}\right)$, and diphthamide formed in EF-2. In other tRNA modification, the acp transfer reaction is involved in the synthesis of wybutosine $(\mathrm{yW})$, a hypermodified nucleoside present in eukaryotic tRNA ${ }^{\text {Phe16. The }}$
SAM-dependent class I methyltransferase TYW2 catalyzes the acp transfer reaction and synthesizes $\mathrm{yW}-86$, a precursor of $\mathrm{yW}^{40,60}$. However, TDD superfamily proteins do not have sequence homology with these acp-transferases ${ }^{49}$. According to the crystal structure of archaeal Tsr3, which is a member of TDD superfamily protein ${ }^{28}$, it has a SPOUT-class RNA methyltransferase fold. The closest structural homolog of the TDD superfamily is a tRNA methyltransferase Trm10. Further structural studies of TapT and DTWD1/2 are necessary to reveal the molecular basis of tRNA substrate recognition and $\operatorname{acp}^{3} \mathrm{U}$ formation.

Measurement of melting temperature revealed that a single acp ${ }^{3} \mathrm{U} 47$ conferred thermal stability to tRNA, increasing the $T_{\mathrm{m}}$ value by $3^{\circ} \mathrm{C}$. In the tertiary structures of tRNAs ${ }^{33-35}$, the acp group of the modification does not interact with any other residues but resides in close vicinity to the backbone of the T-stem. We speculated that the acp group might stabilize the local conformation of tRNA by interacting with the T-stem via magnesium ion or water ${ }^{31}$. In addition, the acp group at the $\mathrm{N} 3$ atom position hinders Watson-Crick base pairing and may destabilize some metastable structures so as to ensure correct folding of tRNA, similarly to the function of $\mathrm{m}^{1} \mathrm{~A} 9^{61}$.

Strikingly, the E. coli $\triangle \operatorname{tap} T$ strain exhibited a small colony phenotype associated with motility defect under continuous heat stress. Because ectopic expression of tapT did not restore the phenotype, we speculated that genomic mutations in swarmingrelated genes might be involved. Curiously, translation stress in $E$. coli strains bearing mutations in tRNA or the tRNA-modifying enzyme causes mutator phenotypes ${ }^{62,63}$. These facts prompted us to speculate that loss of $\mathrm{acp}^{3} \mathrm{U}$ in the $\Delta$ tapT strain contributes to the small colony phenotype mediated by the TSM pathway ${ }^{51}$. Unexpectedly, however, $\Delta t a p T$ strain did not exhibit any mutator phenotypes under continuous heat stress conditions, indicating that neither error-prone replication nor aberrant DNA repairing is involved in this phenotype. Dysregulation of transposable elements are another possible source of genome instability ${ }^{64}$, but this should be investigated in future studies.

To investigate the phylogenetic distribution of the TDD family, we generated a phylogenetic tree of organisms possessing or lacking each homolog (Supplementary Fig. 8a). TSR3 orthologs predominated in eukaryotes and archaea, not in bacteria. Among the eukaryotes, DTWD1 orthologs are only present in vertebrates, insects, nematodes, and protists, but not in fungi and plants. DTWD2 orthologs are divided into two subfamilies, eukaryotic and bacterial (TapT). Eukaryotic DTWD2 orthologs are present in vertebrates, protists, and plants, whereas bacterial DTWD2 (TapT) orthologs are present in Bacteroidetes, Proteobacteria, Actinobacteria, Cyanobacteria, and Firmicutes, as well as in vertebrates, plants, and protists. We constructed multiple alignment of DTWD1 and DTWD2 homologs from representative organisms, and draw a phylogenetic tree using the maximum likelihood method (Supplementary Fig. 8b). DTWD1 orthologs form a small subfamily, clearly separated from the larger DTWD2 family with $100 \%$ bootstrap probability. This tree clearly reveals two major subfamilies of DTWD2: eukaryotic DTWD2, which is responsible for $\operatorname{acp}^{3} U$ formation in the $D$-loop; and bacterial DTWD2 (TapT), which is responsible for $\operatorname{acp}^{3} U$ formation in the $\mathrm{V}$-loop.

T. brucei tRNA ${ }^{\text {Lys }}$ contains $\mathrm{acp}^{3} \mathrm{U} 20$ and $\mathrm{acp}^{3} \mathrm{D} 47^{21}$. Consistent with this finding, one DTWD1 ortholog (Tb09.244.2810) and one DTWD2 ortholog (Tb927.3.4690) are encoded in the T. brucei genome $\mathrm{e}^{49}$, indicating that these proteins are responsible for forming acp ${ }^{3} \mathrm{U} 20$ and $\mathrm{acp}^{3} \mathrm{D} 47$, respectively.

Recently, comprehensive analyses of Vibrio cholerae tRNAs identified $\mathrm{acp}^{3} \mathrm{U}$ and its derivative at positions $20 \mathrm{~b}, 46$, and $47^{65}$. Curiously, the Vibrio cholerae genome encodes three DTWD2 
a

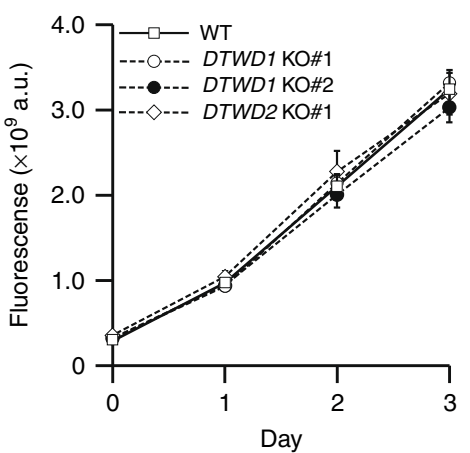

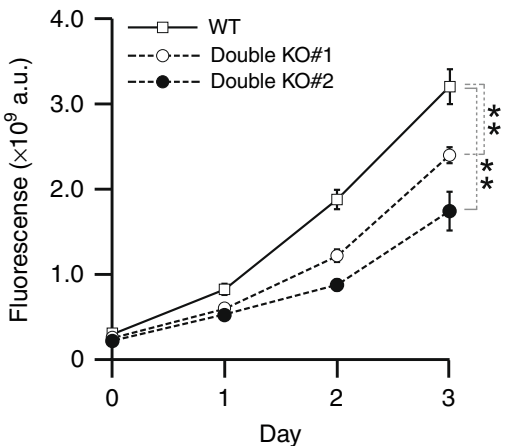

b
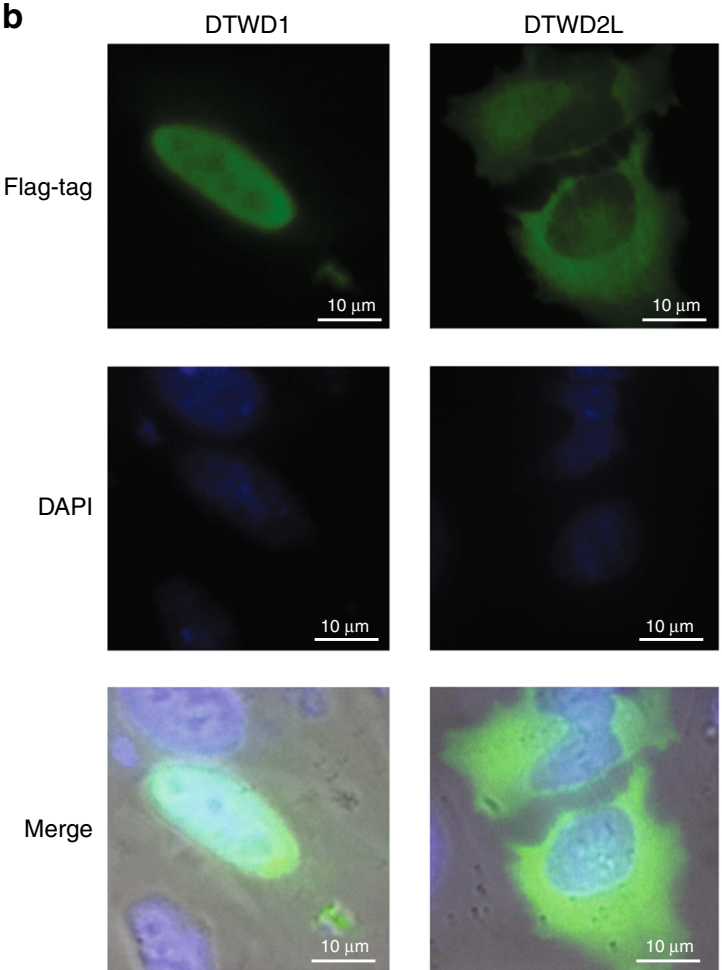
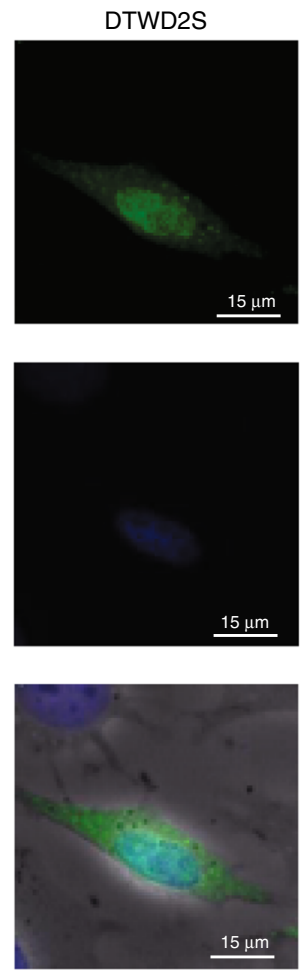

Fig. 5 Physiological role and subcellular localization of DTWD1 and DTWD2. a Growth curves of WT HEK293T (open square), DTWD1 KO\#1 (open circle), DTWD1 KO\#2 (closed circle) and DTWD2 KO (open rhombus) strains (left panel), and WT HEK293T (open square), DTWD1/DTWD2 doubleKO\#1 (open circle) and DTWD1/DTWD2 double-KO\#2 (closed circle) strains (right panel), cultured in DMEM medium. Fluorescence was calculated as the average values of biological replicates $(n=6)$, with s.d. ${ }^{* \star} P<8.1 \times 10^{-6}$ by Student's $t$ test. Source data are provided as a Source Data file. $\mathbf{b}$ Subcellular localization of DTWD1 (left panels), DTWD2L (middle panels), and DTWD2S (right panels) in HeLa cells immunostained with an anti-FLAG antibody (green). Nuclei were stained with DAPI (blue). Images were superimposed to generate the merged panels.

paralogs (VC1533, VC0131, and VC1980). According to the phylogenetic analysis (Supplementary Fig. 8b), VC1533 is a bacterial DTWD2 (TapT) ortholog that is assumed to be responsible for $\mathrm{acp}^{3} \mathrm{U} 47$ formation. Given that VC0131 branches from the root of the bacterial DTWD2 (TapT) subfamily, this homolog might be responsible for acp ${ }^{3} \mathrm{U} 46$ formation. VC1980 forms a unique subfamily with other bacterial orthologs near the eukaryotic DTWD2 subfamily. Considering that eukaryotic DTWD2 is responsible for acp ${ }^{3} \mathrm{U} 20$ a formation, we speculated that the VC1980 subfamily is responsible for acp ${ }^{3} \mathrm{U} 20 \mathrm{~b}$ formation. Pseudomonas species contain two DTWD2 paralogs (Supplementary Fig. 8b). P. aeruginosa PA3606 and PA1424 belong to the bacterial TapT and VC1980-like subfamilies, respectively, and might be responsible for acp ${ }^{3} U 47$ and $a c p^{3} U 20 b$ formation.

In plants, acp ${ }^{3} \mathrm{U} 20 \mathrm{a}$ and $\mathrm{acp}^{3} \mathrm{U} 20 \mathrm{~b}$ are found in nuclearencoded tRNAs, while acp ${ }^{3} \mathrm{U} 47$ is found in tRNAs encoded in plastid and mitochondrial $\mathrm{DNAs}^{20}$. Arabidopsis thaliana has three paralogs of DTWD2 (Supplementary Fig. 8b). AT2G41750 is a typical eukaryotic DTWD2 responsible for $\mathrm{acp}^{3} \mathrm{U} 20$ a formation. AT1G03687, along with other plant orthologs, forms a subfamily closely related to the bacterial DTWD2 (TapT) subfamily, and this paralog is predicted to localize in chloroplasts, implying that it is responsible for $\mathrm{acp}^{3} \mathrm{U} 47$ formation in plastid tRNAs. AT5G54880, along with other plant orthologs, forms a subfamily near the VC1980-like subfamily, implying that this paralog is required for $\mathrm{acp}^{3} \mathrm{U} 20 \mathrm{~b}$ formation. Experimental verification is necessary to confirm these speculations.

Double knockout of DTWD1 and DTWD2 caused severe growth defects in culture cells, indicating the physiological importance of $\mathrm{acp}^{3} \mathrm{U}$ modification. Although the functional roles of this modification remain elusive, it has been suggested that acp ${ }^{3} \mathrm{U} 20 \mathrm{a}$ can coordinate $\mathrm{Mg}^{2+}$, thereby stabilizing the local conformation of $\mathrm{tRNA}^{31}$. The stabilization of tRNAs by acp ${ }^{3} \mathrm{U} 20$ / 20a may lead to efficient translation and rapid growth. According 
to the Genotype-Tissue Expression project (Supplementary Fig. 9a, b) ${ }^{66}, D T W D 1$ and $D T W D 2$ are differentially expressed in various tissues, indicating that the modification frequencies of $\mathrm{acp}^{3} \mathrm{U} 20 / 20$ a might vary. Intriguingly, the two isoforms of DTWD2 exhibited different subcellular localization: DTWD2L and DTWD2S predominantly localized in the cytoplasm and nucleus, respectively. The expression levels of these two isoforms also varied among tissues (Supplementary Fig. 9b). Although expression of DTWD2L is low in many tissues, it is upregulated in others, including testis (Supplementary Fig. 9b). Because the different subcellular localizations of the two isoforms might affect tRNA maturation, tissue-specific isoform expression implies that $\operatorname{acp}^{3} \mathrm{U}$ modification is biologically important.

Loss of tRNA modification results in pathological consequences. We previously reported that human mitochondrial diseases are caused by hypomodification of mitochondrial tRNAs $^{67,68}$ and proposed "RNA modopathy" as a distinct category of human diseases ${ }^{69}$. Recent whole-exome sequencing analyses identified loss-of-function mutations in many genes responsible for tRNA modifications from patients with a wide range of diseases, including neurological disorders, cancers, and diabetes $58,70-73$. DTWD1 is a target of p53 and works as a tumor suppressor gene for gastric cancer ${ }^{74}$ and is downregulated in some cancer cell line. HDAC3 regulates p53-mediated expression of DTWD1. In primary melanomas, high expression of DTWD1 mediated by HIF-1a correlates with poor prognosis and shorter disease-free status ${ }^{75}$. Mutation in DTWD1 is also a risk factor for bipolar disease ${ }^{76}$. Copy number variation in the genome region containing DTWD2 has been observed in primary open-angle glaucoma ${ }^{77}$. Further studies will be necessary to understand the molecular pathogenesis of human diseases associated with loss of $a_{c p}^{3} \mathrm{U}$ modification in tRNAs.

\section{Methods}

Bacterial strains, media, and plasmid construction. $\Delta y f i P$ (JW5409) or $\triangle y a g A$ (JW0260) with kanamycin resistance markers (Keio collection) ${ }^{78}$ and their parental E. coli strain BW25113 (obtained from the Genetic Stock Research Center, National Institute of Genetics, Japan) were cultivated in LB medium with vigorous shaking. A. ferrooxidans (obtained from the Biological Resource Center, National Institute of Technology and Evolution, Japan) was cultured in $0.5 \mathrm{~g} / \mathrm{L} \mathrm{MgSO}_{4} \cdot 7 \mathrm{H}_{2} \mathrm{O}, 0.4 \mathrm{~g} / \mathrm{L}$ $\left(\mathrm{NH}_{4}\right)_{2} \mathrm{SO}_{4}, 0.2 \mathrm{~g} / \mathrm{L} \mathrm{K} \mathrm{HPO}_{4}, 0.1 \mathrm{~g} / \mathrm{L} \mathrm{KCl}, 10 \mathrm{mg} / \mathrm{L} \mathrm{FeSO}{ }_{4} \cdot 7 \mathrm{H}_{2} \mathrm{O}$, and $0.025 \%$ yeast extract (adjusted to $\mathrm{pH} 2.0$ with $2 \mathrm{M} \mathrm{H}_{2} \mathrm{SO}_{4}$ ) at $45^{\circ} \mathrm{C}^{79}$. Synechocystis sp. PCC 6803 (kindly provided by K. Sonoike of Waseda University, Japan) was cultured with BG11 medium ${ }^{80}$ under LED light at $34^{\circ} \mathrm{C}$. T. thermophilus $\mathrm{HB} 27$ (kindly provided by N. Shigi of AIST, Japan) was cultured in rich medium containing $0.8 \%$ polypeptone, $0.4 \%$ yeast extract, and $0.3 \% \mathrm{NaCl}$ (adjusted to $\mathrm{pH} 7.5$ with $1 \mathrm{M} \mathrm{HCl}$ ) at $70^{\circ} \mathrm{C}$ rich medium as previously described ${ }^{81}$. B. subtilis str. 168 (kindly provided by A. Soma of Chiba University, Japan) was cultured in $\mathrm{LB}$ medium at $37^{\circ} \mathrm{C}^{82} . M$. mobile 163K (kindly provided by M. Miyata of Osaka City University, Japan) was grown in Aluotto medium ( $\mathrm{pH} 7.8$ ), consisting of $2.1 \%$ heart infusion broth (Difco), $0.56 \%$ yeast extract, $10 \%$ horse serum (inactivated at $56^{\circ} \mathrm{C}$ ), and $0.005 \%$ ampicillin at $25^{\circ} \mathrm{C}^{83}$.

To construct a plasmid encoding $y f i P$ gene for complementation, the open reading frame of $y f i P$ with promoter region was PCR-amplified from the E. coli genome using a set of primers listed in Supplementary Table 1. Amplified products were inserted into the HindIII/EcoRI sites of the pMW118 vector (Invitrogen).

\section{Human cell culture and measurement of cell proliferation. HEK293T (CRL-} 11268, ATCC) and Hela (CCL-2, ATCC) cells were cultured in high-glucose DMEM (D5796; Sigma) supplemented with 5\% FBS (Gibco) and 1\%

penicillin-streptomycin (Fujifilm Wako Pure Chemical Corporation), at $37^{\circ} \mathrm{C}$ in a humidified atmosphere containing $5 \% \mathrm{CO}_{2}$. To compare cell proliferation, WT HEK293T cells and a series of knockout cells were seeded into 96-well plates $(4.0 \times$ $10^{3}$ cells per well). Each day, a 1/10th volume of $1 \mathrm{mM}$ resazurin solution in PBS was added to each well, and the cells were incubated for $3 \mathrm{~h}$. The fluorescence of reduced resazurin was measured using a fluorescence microplate reader (SpectraMax Paradigm MultiMode Detection Platform; Molecular Devices) with excitation/emission at $560 / 590 \mathrm{~nm}$.

Construction of human DTWD1/DTWD2 KO cell lines. DTWD1 and DTWD2 single- and double-KO cells were generated using the CRISPR-Cas9 system, as described ${ }^{69,84}$. Two sgRNAs targeting exon 1 of the DTWD1 gene, and two
sgRNAs targeting exon 2 or 3 of the DTWD2 gene, were designed. Sense and antisense oligonucleotides for the sgRNAs (Supplementary Table 1) were cloned into the pX330 vector (Addgene plasmid 42230) ${ }^{85}$. HEK293T cells $\left(1.0 \times 10^{5}\right.$ cells per plate) were transfected with the vector bearing the sgRNA sequence, along with pEGFP-N1 (Clontech) and modified pLL3.7 vectors harboring a puromycin resistance gene. One day after transfection, the cells were seeded at low density and selected with $1 \mu \mathrm{g} / \mathrm{mL}$ puromycin. The KO cell lines were selected by confirming the frameshift mutations in the target region. DTWD1/DTWD2 double-KO cells were generated from the DTWD1 single-KO cells.

RNA extraction and isolation of individual tRNAs. Total RNAs of $E$. coli were extracted as described ${ }^{86}$. Total RNAs of A. ferrooxidans, Synechocystis sp. PCC 6803 , T. thermophilus HB27, B. subtilis str.168, and M. mobile $163 \mathrm{~K}$ were extracted using the acidic phenol method as described ${ }^{79,83}$. Total RNAs were extracted from HEK293T cells using TriPure Isolation Reagent (Roche Life Science).

Individual tRNAs were isolated by reciprocal circulating chromatography as described ${ }^{87}$ using the $5^{\prime}$-terminal ethylcarbamate amino-modified DNA probes (Sigma Aldrich Japan) listed in Supplementary Table 1.

Mass spectrometric analysis. For nucleoside analysis of E. coli, total RNAs $(1.6 \mu \mathrm{g})$ were digested with $0.8 \mathrm{U}$ nuclease P1 (FUJIFILM Wako Pure Chemical) at $37^{\circ} \mathrm{C}$ for $60 \mathrm{~min}$. The digests were adjusted to $50 \mathrm{mM}$ ammonium bicarbonate ( $\mathrm{pH} 8.2$ ), followed by addition of $0.2 \mathrm{U}$ phosphodiesterase I (Worthington) and incubated at $37^{\circ} \mathrm{C}$ for $60 \mathrm{~min}$, then $0.2 \mathrm{U}$ of bacterial alkaline phosphatase (BAP, from E. coli C75, FUJIFILM Wako Pure Chemical) was added, and incubated at $37^{\circ} \mathrm{C}$ for $60 \mathrm{~min}$, as described ${ }^{86}$. Nucleosides were dissolved in $90 \%$ acetonitrile ( $10 \%$ water), and subjected to a hydrophilic interaction LC (ZIC-cHILIC, $3 \mu \mathrm{m}$ particle size, $2.1 \times 150 \mathrm{~mm}$, Merck) coupled with ESI-MS on a Q Exactive hybrid Quadrupole-Orbitrap mass spectrometer (Thermo Fisher Scientific), equipped with an ESI source and an Ultimate 3000 liquid chromatography system (Thermo Fisher Scientific) ${ }^{88}$. The mobile phase consisted of $5 \mathrm{mM}$ ammonium acetate ( $\mathrm{pH} 5.3$ ) (solvent A) and acetonitrile (solvent $\mathrm{B}$ ), and the nucleosides were chromatographed with a flow rate of $100 \mu \mathrm{L} / \mathrm{min}$ in a multistep linear gradient; $90-40 \%$ B from 0 to $30 \mathrm{~min}, 40 \% \mathrm{~B}$ for $10 \mathrm{~min}$, and then initialized to $90 \% \mathrm{~B}$. Proton adducts of nucleosides were scanned in a positive polarity mode over an $\mathrm{m} / \mathrm{z}$ range of 103-700. Xcalibur 3.0.63 (Thermo Fisher Scientific) was used for the system operation.

Nucleoside analyses for comparative genomics were performed by reversephase chromatography/ESI-MS. Total RNAs $(16-160 \mu \mathrm{g})$ were digested in a reaction mixture containing with $20 \mathrm{mM}$ ammonium acetate ( $\mathrm{pH} 5.3$ ), $0.04-0.08 \mathrm{U}$ $\mathrm{BAP}$, and $0.05-0.1 \mathrm{U}$ nuclease P1, and subjected to InertSil ODS-3 (5 $\mu \mathrm{m}$ particle size, $2.1 \times 250 \mathrm{~mm}$, GL sciences) or SunShell C18 $(2.6 \mu \mathrm{m}$ particle size, $2.1 \times 150$ $\mathrm{mm}$, ChromaNik Technologies) column, then analyzed by an LCQ Advantage iontrap mass spectrometer (Thermo Fisher Scientific) with an ESI source and an HP1100 liquid chromatography system (Agilent Technologies) ${ }^{79}$ or a Q Exactive hybrid Quadrupole-Orbitrap mass spectrometer (Thermo Fisher Scientific) equipped with an ESI source and an Ultimate 3000 liquid chromatography system (Thermo Fisher Scientific) ${ }^{88}$. The mobile phase consisted of $5 \mathrm{mM}$ ammonium acetate ( $\mathrm{pH}$ 5.3) (solvent $\mathrm{A}$ ) and acetonitrile (solvent B). Xcalibur 2.0 SUR1 (Thermo Fisher Scientific) was used for operation of the LCQ Advantage system,

For RNA fragment analysis, the isolated tRNAs ( 2 pmol) were digested with 10 $\mathrm{U}$ of RNase $\mathrm{T}_{1}$ in $20 \mathrm{mM}$ ammonium acetate ( $\mathrm{pH} \mathrm{5.3)}$ ) at $37^{\circ} \mathrm{C}$ for $60 \mathrm{~min}$. The digests were mixed with $1 / 10$ vol. of $0.1 \mathrm{M}$ triethylamine acetate $(\mathrm{pH}$ 7.0) and subjected to LC/nano ESI-MS on an LTQ Orbitrap mass spectrometer (Thermo Fisher Scientific) equipped with a splitless nanoflow high-performance LC (nanoHPLC) system (DiNa, KYA Technologies) using a nano-LC trap column (C18, $0.1 \times 0.5 \mathrm{~mm}, \mathrm{KYA}$ Technologies) and a capillary column (HiQ Sil C18W-3, $0.1 \times$ $100 \mathrm{~mm}, \mathrm{KYA}$ Technologies) as described ${ }^{37}$. Digested fragments were separated for $40 \mathrm{~min}$ at a flow rate of $300 \mathrm{~nL} / \mathrm{min}$ by capillary LC using a linear gradient from 2 to $100 \%$ solvent $\mathrm{B}(\mathrm{v} / \mathrm{v})$ in a solvent system consisting of $0.4 \mathrm{M} 1,1,1,3,3,3$ hexafluoro-2-propanol (HFIP) ( $\mathrm{pH}$ 7.0) (solvent A) and 0.4 M HFIP (pH 7.0) in $50 \%$ methanol (solvent $\mathrm{B})$. The eluent was ionized by ESI source in a negative polarity and scanned over an $\mathrm{m} / \mathrm{z}$ range of 600-2000. Xcalibur 2.0.7 (Thermo Fisher Scientific) was used for the system operation.

The LC/MS data were analyzed using Xcalibur Qual browser (Thermo Fisher Scientific). In nucleoside analysis, the peak of modified nucleoside was normalized by the peak area of the unmodified nucleoside. In RNA fragment analysis, the peak of each fragment was normalized by the sum of modified and unmodified fragments. Mongo Oligo Mass Calculator v2.08 (https://mods.rna.albany.edu/ masspec/Mongo-Oligo) was used for assignment of the product ions in CID spectra.

Expression and purification of recombinant protein. To construct an expression plasmid for TapT recombinant protein, the open reading frame of $y$ fiP was PCRamplified from the E. coli genome using the primers listed in Supplementary Table 1 . The amplified products were inserted into the NdeI/XhoI sites of the pET28a vector (Invitrogen) to yield pET-TapT, which expresses the N-terminally His-tagged TapT protein. The E. coli BL21(DE3) strain was transformed with the plasmid and cultured at $37^{\circ} \mathrm{C}$. When the absorbance at $600 \mathrm{~nm}\left(A_{600}\right)$ reached 0.5 , 
isopropyl- $\beta$-D-thiogalactopyranoside was added to a final concentration of $0.1 \mathrm{mM}$ for inducing expression, and the cells were cultured at $18^{\circ} \mathrm{C}$ for an additional $24 \mathrm{~h}$. The cells were harvested and lysed by sonication in lysis buffer $20 \mathrm{mM}$ HEPES$\mathrm{KOH}(\mathrm{pH} 7.5), 500 \mathrm{mM} \mathrm{KCl}, 6 \mathrm{mM} \beta$-mercaptoethanol ( $\beta-\mathrm{Me})$, and $1 \mathrm{mM}$ phenylmethanesulfonyl fluoride]. The soluble recombinant protein was purified using a HisTrap HP column (GE Healthcare) and anion exchange chromatography (Mono-Q HR 5/5, GE Healthcare) using the AKTA Purifier 10 system (GE Healthcare). The purified protein was dialyzed against a buffer consisting of $20 \mathrm{mM}$ HEPES-KOH (pH 7.5), $200 \mathrm{mM} \mathrm{KCl,} 6 \mathrm{mM} \beta-\mathrm{Me}$, and $10 \%$ glycerol, and then stored at $-80^{\circ} \mathrm{C}$.

In vitro reconstitution of $\operatorname{acp}^{3} \mathbf{U}$ by TapT. For the substrates, we isolated tRNAMet from the $\Delta \operatorname{tap} T$ strain by reciprocal circulating chromatography ${ }^{87}$. The tRNA ${ }^{\text {Met }}$ transcript was prepared by in vitro transcription using T7 RNA polymerase, essentially as described ${ }^{89}$. The synthetic DNAs used for template construction by PCR are listed in Supplementary Table 1. In vitro transcription was performed at $37^{\circ} \mathrm{C}$ overnight in a reaction mixture containing $40 \mathrm{mM}$ Tris- $\mathrm{HCl}(\mathrm{pH} 7.5), 24 \mathrm{mM} \mathrm{MgCl}_{2}, 5 \mathrm{mM}$ DTT, $2.5 \mathrm{mM}$ spermidine, $0.01 \%$ Triton X-100, $30 \mathrm{nM}$ DNA template, $2 \mathrm{mM}$ GTP, $2 \mathrm{mM}$ CTP, $2 \mathrm{mM}$ UTP, $2 \mathrm{mM}$ ATP, and $10 \mathrm{mM}$ GMP. Subsequently, the reaction mixture was subjected to phenol-chloroform extraction and desalted by passing through a PD10 columns (GE Healthcare). The transcribed tRNA was purified by running on $10 \%$ denaturing polyacrylamide gel electrophoresis (PAGE).

In vitro reconstitution of $\mathrm{acp}^{3} \mathrm{U}$ modification was performed at $37^{\circ} \mathrm{C}$ for $2 \mathrm{~h}$ in a reaction mixture containing $20 \mathrm{mM}$ HEPES- $\mathrm{KOH}$ (pH 7.5), $10 \mathrm{mM} \mathrm{MgCl} 2,100$ $\mathrm{mM} \mathrm{NaCl}, 1 \mathrm{mM}$ DTT, $1 \mathrm{mM}$ SAM, $8.5 \mu \mathrm{M}$ tRNA ${ }^{\mathrm{Met}}$, and $0.75 \mu \mathrm{M}$ recombinant TapT. The tRNA product was digested with RNase $\mathrm{T}_{1}$ (Thermo Fisher Scientific) and subjected to LC/MS analysis as described above.

Measurement of thermal stability of tRNAs. The tRNA $(2.5 \mu \mathrm{g})$ was dissolved in degassed buffer containing $20 \mathrm{mM}$ HEPES-KOH (pH 7.5), $150 \mathrm{mM} \mathrm{NaCl}$, and 6 $\mathrm{mM} \mathrm{MgCl}$, and incubated at $75^{\circ} \mathrm{C}$ for $5 \mathrm{~min}$, followed by cooling at room temperature for annealing. A UV-Vis spectrophotometer (V-630; JASCO) equipped with an 8 multi-quartz micro cell array (path length: $10 \mathrm{~mm}$; JASCO) was used to monitor the hyperchromicity of tRNA. The temperature gradient was as follows: $25^{\circ} \mathrm{C}$ for $30 \mathrm{~s}$, and then ramped at $5{ }^{\circ} \mathrm{C} / \mathrm{min}$ to $40^{\circ} \mathrm{C}$ and held for $5 \mathrm{~min}$, and ramped at $0.5^{\circ} \mathrm{C} / \mathrm{min}$ to $90^{\circ} \mathrm{C}$.

Morphological analysis under heat-stressed conditions. E. coli strains were cultivated in $5 \mathrm{~mL} \mathrm{LB}$ medium with vigorous shaking at $37^{\circ} \mathrm{C}$ or $42^{\circ} \mathrm{C}$ for $16-24 \mathrm{~h}$ until reaching the late stationary phase. Then, cells were diluted by a factor of $10^{6}$ or $10^{7}$ so that the concentration was suitable for counting the colony numbers. One hundred microliters of the diluted cells were spread on LB agar plate and cultured at $37^{\circ} \mathrm{C}$ overnight. In parallel, $2.5 \mu \mathrm{L}$ harvested cells was inoculated into $5 \mathrm{~mL}$ fresh LB medium and cultured at the same temperature. The process described above was repeated for 5-7 days. The frequency of small colonies appearing on the plate was calculated by counting the number of normal and small colonies on each plate.

Motility assay. The motility assay was performed basically as described ${ }^{90}$. Precultured $E$. coli cells were diluted to $0.01 A_{600}$, and then $2 \mu \mathrm{L}$ diluted cells was inoculated at the center of the soft agar plate (1\% tryptone, $1 \% \mathrm{NaCl}$, and $0.3 \%$ agar). The plates were cultured at $30^{\circ} \mathrm{C}$ for $16 \mathrm{~h}$. The swarming diameter of each strain was averaged from five different sets of measurements.

Mutator assay. The mutator assay was carried out basically as described ${ }^{52,91,92}$. In addition to the $\triangle t a p T$ strain, $\triangle m u t S, \triangle m i a A, \triangle y a g A$ strains were used as the strong mutator, mild mutator, and negative controls, respectively. E. coli cells were cultivated at $42^{\circ} \mathrm{C}$ for 5 days and then plated on LB plates at every passage as described in the morphological analysis section. At every passage, adequate amounts of the harvested cells were spread on LB agar plates containing $80 \mu \mathrm{g} / \mathrm{mL}$ nalidixic acid and cultured at $37^{\circ} \mathrm{C}$ overnight. Mutation rate was calculated from the ratio of colony numbers on the LB plates with and without nalidixic acid.

Northern blotting. Total RNAs $(4 \mu \mathrm{g})$ from WT HEK293T and DTWD1/DTWD2 double-KO cells were dissolved by $10 \%$ denaturing PAGE, stained with SYBR safe (Invitrogen), and blotted onto a nylon membrane (Amersham Hybond N+; GE Healthcare) in $0.5 \times$ TBE using a Transblot Turbo apparatus (Bio-Rad). Hybridization was performed essentially according to the manufacturer's instructions (PerfectHyb; TOYOBO) at $42^{\circ} \mathrm{C}$ with 2 pmol of $5^{\prime}-32 \mathrm{P}$-labeled oligonucleotides (Supplementary Table 1) complementary to each target tRNA. The membrane was washed three times with $2 \times$ SSC, dried, and exposed to an imaging plate (BASMS2040; Fujifilm). Radioactivity was visualized using an FLA-7000 imaging analyzer (Fujifilm).

Immunostaining. Hela cells $\left(4.0 \times 10^{5}\right.$ cells per dish) were transfected with the pcDNA-3.1 vectors (Invitrogen) bearing the open reading frame of DTWD1, $D T W D 2 L$, or $D T W D 2 S$, each of which expresses the C-terminally flag-tagged protein. The cells were fixed with $3.7 \%$ formaldehyde, permeabilized with $1 \%$ Triton X-100, and blocked with 20\% Ezblockchemi (ATTO). Immunostaining was performed with anti-DYKDDDDK IgG antibody (1:1000) (014-22383, Wako) for the primary antibody and anti-mouse IgG Alexa fluor 488 (1:1000) (A-11001, Invitrogen) for the secondary antibody. Then, cells were stained with DAPI $(1: 10,000)$. The pictures were taken using DMI 6000 (Leica).

Reporting Summary. Further information on research design is available in the Nature Research Reporting Summary linked to this Article.

\section{Data availability}

A reporting summary for this article is available as a Supplementary Information file. The source data underlying Figs. 2f, 3b-d, and 5a and Supplementary Figs. 3b, 4a, b, and 7a, b are provided as a Source Data file. All data supporting the findings in this study are available from the corresponding author upon reasonable request.

Received: 13 September 2019; Accepted: 11 November 2019; Published online: 05 December 2019

\section{References}

1. Boccaletto, P. et al. MODOMICS: a database of RNA modification pathways. 2017 update. Nucleic Acids Res. 46, D303-D307 (2018).

2. Suzuki, T. in Fine-Tuning of RNA Functions by Modification and Editing. (ed Grosjean, Ed.) 24-69 (Springer-Verlag, NY, 2005).

3. Motorin, Y. \& Helm, M. tRNA stabilization by modified nucleotides. Biochemistry 49, 4934-4944 (2010).

4. Jackman, J. E. \& Alfonzo, J. D. Transfer RNA modifications: nature's combinatorial chemistry playground. Wiley Interdiscip. Rev. RNA 4, 35-48 (2013).

5. Dalluge, J. J., Hashizume, T., Sopchik, A. E., McCloskey, J. A. \& Davis, D. R Conformational flexibility in RNA: the role of dihydrouridine. Nucleic Acids Res. 24, 1073-1079 (1996).

6. Yokoyama, S., Watanabe, K. \& Miyazawa, T. Dynamic structures and functions of transfer ribonucleic acids from extreme thermophiles. Adv. Biophys. 23, 115-147 (1987)

7. Sengupta, R. et al. Modified constructs of the tRNA TPsiC domain to probe substrate conformational requirements of $\mathrm{m}(1) \mathrm{A}(58)$ and $\mathrm{m}(5) \mathrm{U}(54)$ tRNA methyltransferases. Nucleic Acids Res. 28, 1374-1380 (2000).

8. Ladner, J. E. et al. Structure of yeast phenylalanine transfer RNA at $2.5 \mathrm{~A}$ resolution. Proc. Natl Acad. Sci. USA 72, 4414-4418 (1975).

9. Arnez, J. G. \& Steitz, T. A. Crystal structure of unmodified tRNA(Gln) complexed with glutaminyl-tRNA synthetase and ATP suggests a possible role for pseudo-uridines in stabilization of RNA structure. Biochemistry 33, 7560-7567 (1994).

10. Davanloo, P., Sprinzl, M., Watanabe, K., Albani, M. \& Kersten, H. Role of ribothymidine in the thermal stability of transfer RNA as monitored by proton magnetic resonance. Nucleic Acids Res. 6, 1571-1581 (1979).

11. Gregson, J. M. et al. Structure of the archaeal transfer RNA nucleoside $\mathrm{G}^{*}-15$ (2-amino-4,7-dihydro- 4-oxo-7-beta-D-ribofuranosyl-1H-pyrrolo[2,3-d] pyrimidine-5-carboximi dam ide (archaeosine)). J. Biol. Chem. 268, 10076-10086 (1993).

12. Watanabe, K., Oshima, T., Saneyoshi, M. \& Nishimura, S. Replacement of ribothymidine by 5 -methyl-2-thiouridine in sequence GT psi C in tRNA of an extreme thermophile. FEBS Lett. 43, 59-63 (1974).

13. Hori, H. et al. Transfer RNA modification enzymes from thermophiles and their modified nucleosides in tRNA. Microorganisms 6, 110 (2018).

14. Watanabe, K., Oshima, T., Hansske, F. \& Ohta, T. Separation and comparison of 2-thioribothymidine-containing transfer ribonucleic acid and the ribothymidine-containing counterpart from cells of Thermus thermophilus $\mathrm{HB}$ 8. Biochemistry 22, 98-102 (1983).

15. Orita, I. et al. Random mutagenesis of a hyperthermophilic archaeon identified tRNA modifications associated with cellular hyperthermotolerance. Nucleic Acids Res. 47, 1964-1976 (2019).

16. Phizicky, E. M. \& Hopper, A. K. tRNA biology charges to the front. Genes Dev. 24, 1832-1860 (2010).

17. Kimura, S. \& Waldor, M. K. The RNA degradosome promotes tRNA quality control through clearance of hypomodified tRNA. Proc. Natl Acad. Sci. USA 116, 1394-1403 (2019).

18. Friedman, S., Li, H. J., Nakanishi, K. \& Van Lear, G. 3-(3-amino-3-carboxy-npropyl)uridine. The structure of the nucleoside in Escherichia coli transfer ribonucleic acid that reacts with phenoxyacetoxysuccinimide. Biochemistry 13, 2932-2937 (1974)

19. Ohashi, Z., Maeda, M., McCloskey, J. A. \& Nishimura, S. 3-(3-amino-3carboxypropyl)uridine: a novel modified nucleoside isolated from Escherichia coli phenylalanine transfer ribonucleic acid. Biochemistry 13, 2620-2625 (1974). 
20. Jühling, F. et al. tRNAdb 2009: compilation of tRNA sequences and tRNA genes. Nucleic Acids Res. 37, D159-D162 (2009).

21. Krog, J. S. et al. 3-(3-amino-3-carboxypropyl)-5,6-dihydrouridine is one of two novel post-transcriptional modifications in tRNALys(UUU) from Trypanosoma brucei. FEBS J. 278, 4782-4796 (2011).

22. Saponara, A. G. \& Enger, M. D. The isolation from ribonucleic acid of substituted uridines containing alpha-aminobutyrate moieties derived from methionine. Biochim Biophys. Acta 349, 61-77 (1974).

23. Maden, B. E., Forbes, J., de Jonge, P. \& Klootwijk, J. Presence of a hypermodified nucleotide in HeLa cell $18 \mathrm{~S}$ and Saccharomyces carlsbergensis 17 S ribosomal RNAs. FEBS Lett. 59, 60-63 (1975).

24. Sharma, S. \& Lafontaine, D. L. J. View from a bridge': a new perspective on eukaryotic rRNA base modification. Trends Biochem Sci. 40, 560-575 (2015).

25. Kiss, A. M., Jády, B. E., Bertrand, E. \& Kiss, T. Human box H/ACA pseudouridylation guide RNA machinery. Mol. Cell Biol. 24, 5797-5807 (2004).

26. Wurm, J. P. et al. The ribosome assembly factor Nep1 responsible for BowenConradi syndrome is a pseudouridine-N1-specific methyltransferase. Nucleic Acids Res. 38, 2387-2398 (2010).

27. Enger, M. D. \& Saponara, A. G. Incorporation of $14 \mathrm{C}$ from [2-14C] methionine into $18 \mathrm{~s}$ but not $28 \mathrm{~s}$ RNA of Chinese hamster cells. J. Mol. Biol. 33, 319-322 (1968).

28. Meyer, B. et al. Ribosome biogenesis factor Tsr3 is the aminocarboxypropyl transferase responsible for $18 \mathrm{~S}$ rRNA hypermodification in yeast and humans. Nucleic Acids Res. 44, 4304-4316 (2016).

29. Li, Z. et al. Rational extension of the ribosome biogenesis pathway using network-guided genetics. PLoS Biol. 7, e1000213 (2009).

30. Chang, Y. C., Herath, J., Wang, T. H. \& Chow, C. S. Synthesis and solution conformation studies of 3-substituted uridine and pseudouridine derivatives. Bioorg. Med. Chem. 16, 2676-2686 (2008).

31. Stuart, J. W. et al. Structure of the trinucleotide D-acp3U-A with coordinated $\mathrm{Mg} 2+$ demonstrates that modified nucleosides contribute to regional conformations of RNA. Nucleosides Nucleotides 15, 1009-1028 (1996).

32. Friedman, S. The effect of chemical modification of 3-(3-amino-3carboxypropyl)uridine on tRNA function. J. Biol. Chem. 254, 7111-7115 (1979).

33. Rozov, A. et al. Importance of potassium ions for ribosome structure and function revealed by long-wavelength X-ray diffraction. Nat. Commun. 10, 2519 (2019).

34. Arenz, S. et al. A combined cryo-EM and molecular dynamics approach reveals the mechanism of ErmBL-mediated translation arrest. Nat. Commun. 7, 12026 (2016).

35. Brown, A., Fernandez, I. S., Gordiyenko, Y. \& Ramakrishnan, V. Ribosomedependent activation of stringent control. Nature 534, 277-280 (2016).

36. Nishimura, S., Taya, Y., Kuchino, Y. \& Oashi, Z. Enzymatic synthesis of 3-(3amino-3-carboxypropyl)uridine in Escherichia coli phenylalanine transfer RNA: transfer of the 3-amino-acid-3-carboxypropyl group from Sadenosylmethionine. Biochem. Biophys. Res. Commun. 57, 702-708 (1974).

37. Suzuki, T., Ikeuchi, Y., Noma, A. \& Sakaguchi, Y. Mass spectrometric identification and characterization of RNA-modifying enzymes. Methods Enzymol. 425, 211-229 (2007).

38. Sakai, Y., Kimura, S. \& Suzuki, T. Dual pathways of tRNA hydroxylation ensure efficient translation by expanding decoding capability. Nat. Commun. 10, 2858 (2019).

39. Soma, A. et al. An RNA-modifying enzyme that governs both the codon and amino acid specificities of isoleucine tRNA. Mol. Cell 12, 689-698 (2003).

40. Noma, A., Kirino, Y., Ikeuchi, Y. \& Suzuki, T. Biosynthesis of wybutosine, a hyper-modified nucleoside in eukaryotic phenylalanine tRNA. EMBO J. 25, 2142-2154 (2006).

41. Ikeuchi, Y., Shigi, N., Kato, J., Nishimura, A. \& Suzuki, T. Mechanistic insights into sulfur relay by multiple sulfur mediators involved in thiouridine biosynthesis at tRNA wobble positions. Mol. Cell 21, 97-108 (2006).

42. Kimura, S. \& Suzuki, T. Fine-tuning of the ribosomal decoding center by conserved methyl-modifications in the Escherichia coli $16 \mathrm{~S}$ rRNA. Nucleic Acids Res 38, 1341-1352 (2010).

43. Ito, S. et al. A single acetylation of $18 \mathrm{~S}$ rRNA is essential for biogenesis of the small ribosomal subunit in Saccharomyces cerevisiae. J. Biol. Chem. 289, 26201-26212 (2014).

44. Akichika, S. et al. Cap-specific terminal N (6)-methylation of RNA by an RNA polymerase II-associated methyltransferase. Science 363, eaav008 (2019).

45. Kaneko, T. et al. Wobble modification differences and subcellular localization of tRNAs in Leishmania tarentolae: implication for tRNA sorting mechanism. EMBO J. 22, 657-667 (2003).

46. Uchiyama, I., Mihara, M., Nishide, H. \& Chiba, H. MBGD update 2015: microbial genome database for flexible ortholog analysis utilizing a diverse set of genomic data. Nucleic Acids Res. 43, D270-D276 (2015).

47. Keseler, I. M. et al. The EcoCyc database: reflecting new knowledge about Escherichia coli K-12. Nucleic Acids Res. 45, D543-D550 (2017).
48. Ovchinnikov, S. et al. Large-scale determination of previously unsolved protein structures using evolutionary information. eLife 4, e09248 (2015).

49. Burroughs, A. M. \& Aravind, L. Analysis of two domains with novel RNAprocessing activities throws light on the complex evolution of ribosomal RNA biogenesis. Front. Genet. 5, 424 (2014).

50. Zhao, J., Leung, H. E. \& Winkler, M. E. The miaA mutator phenotype of Escherichia coli K-12 requires recombination functions. J. Bacteriol. 183, 1796-1800 (2001).

51. Humayun, M. Z. SOS and Mayday: multiple inducible mutagenic pathways in Escherichia coli. Mol. Microbiol. 30, 905-910 (1998).

52. Luria, S. E. \& Delbruck, M. Mutations of bacteria from virus sensitivity to virus resistance. Genetics 28, 491-511 (1943).

53. Fishel, R. Mismatch repair. J. Biol. Chem. 290, 26395-26403 (2015).

54. Johnson, G. D., Pirtle, I. L. \& Pirtle, R. M. The nucleotide sequence of tyrosine tRNAQ* psi A from bovine liver. Arch. Biochem. Biophys. 236, 448-453 (1985).

55. Roe, B. A. et al. Comparison of rat liver and Walker 256 carcinosarcoma tRNAs. Nucleic Acids Res. 6, 673-688 (1979).

56. Clark, W. C., Evans, M. E., Dominissini, D., Zheng, G. \& Pan, T. tRNA base methylation identification and quantification via high-throughput sequencing. RNA 22, 1771-1784 (2016).

57. Kato, T. et al. A novel human tRNA-dihydrouridine synthase involved in pulmonary carcinogenesis. Cancer Res. 65, 5638-5646 (2005).

58. de Crécy-Lagard, V. et al. Matching tRNA modifications in humans to their known and predicted enzymes. Nucleic Acids Res. 47, 2143-159 (2019).

59. Lin, H. S-adenosylmethionine-dependent alkylation reactions: when are radical reactions used? Bioorg. Chem. 39, 161-170 (2011).

60. Umitsu, M. et al. Structural basis of AdoMet-dependent aminocarboxypropyl transfer reaction catalyzed by tRNA-wybutosine synthesizing enzyme, TYW2. Proc. Natl Acad. Sci. USA 106, 15616-15621 (2009).

61. Helm, M., Giegé, R. \& Florentz, C. A Watson-Crick base-pair-disrupting methyl group $\left(\mathrm{m}^{1} \mathrm{~A} 9\right)$ is sufficient for cloverleaf folding of human mitochondrial tRNALys. Biochemistry 38, 13338-13346 (1999).

62. Connolly, D. M. \& Winkler, M. E. Structure of Escherichia coli K-12 miaA and characterization of the mutator phenotype caused by miaA insertion mutations. J. Bacteriol. 173, 1711-1721 (1991)

63. Slupska, M. M., Baikalov, C., Lloyd, R. \& Miller, J. H. Mutator tRNAs are encoded by the Escherichia coli mutator genes mutA and mutC: a novel pathway for mutagenesis. Proc. Natl Acad. Sci. USA 93, 4380-4385 (1996).

64. Darmon, E. \& Leach, D. R. Bacterial genome instability. Microbiol. Mol. Biol. Rev. 78, 1-39 (2014).

65. Kimura, S., Dedon, P. C. \& Waldor, M. K. Surveying the landscape of tRNA modifications by combining tRNA sequencing and RNA mass spectrometry. Preprint at https://www.biorxiv.org/content/10.1101/723049v1 (2019).

66. Carithers, L. J. et al. A novel approach to high-quality postmortem tissue procurement: the GTEx Project. Biopreserv. Biobank. 13, 311-319 (2015).

67. Suzuki, T., Nagao, A. \& Suzuki, T. Human mitochondrial tRNAs: biogenesis, function, structural aspects, and diseases. Annu. Rev. Genet. 45, 299-329 (2011).

68. Suzuki, T., Nagao, A. \& Suzuki, T. Human mitochondrial diseases caused by lack of taurine modification in mitochondrial tRNAs. Wiley Interdiscip. Rev. RNA 2, 376-386 (2011)

69. Asano, K. et al. Metabolic and chemical regulation of tRNA modification associated with taurine deficiency and human disease. Nucleic Acids Res. 46, $1565-1583$ (2018).

70. Zhou, Z., Sun, B., Huang, S., Jia, W. \& Yu, D. The tRNA-associated dysregulation in diabetes mellitus. Metabolism 94, 9-17 (2019).

71. Hawer, $\mathrm{H}$. et al. Roles of elongator dependent tRNA modification pathways in neurodegeneration and cancer. Genes 10, 19 (2018).

72. Ramos, J. \& Fu, D. The emerging impact of tRNA modifications in the brain and nervous system. Biochim. Biophys. Acta Gene Regul. Mech. 1862, 412-428 (2018).

73. Pereira, M. et al. Impact of tRNA modifications and tRNA-modifying enzymes on proteostasis and human disease. Int J. Mol. Sci. 19, E3738 (2018).

74. Ma, Y. et al. Histone deacetylase 3 inhibits new tumor suppressor gene DTWD1 in gastric cancer. Am. J. Cancer Res. 5, 663-673 (2015).

75. Loftus, S. K. et al. Hypoxia-induced HIFla targets in melanocytes reveal a molecular profile associated with poor melanoma prognosis. Pigment Cell Melanoma Res. 30, 339-352 (2017).

76. Budde, M. et al. Efficient region-based test strategy uncovers genetic risk factors for functional outcome in bipolar disorder. Eur. Neuropsychopharmacol. 29, 156-170 (2019).

77. Davis, L. K. et al. Copy number variations and primary open-angle glaucoma. Investig. Ophthalmol. Vis. Sci. 52, 7122-7133 (2011).

78. Baba, T. et al. Construction of Escherichia coli K-12 in-frame, single-gene knockout mutants: the Keio collection. Mol. Syst. Biol. 2, 2006.0008 (2006).

79. Miyauchi, K., Kimura, S. \& Suzuki, T. A cyclic form of N6threonylcarbamoyladenosine as a widely distributed tRNA hypermodification. Nat. Chem. Biol. 9, 105-111 (2013). 
80. Rippka, R., Deruelles, J., Waterbury, J. B., Herdman, M. \& Stanier, R. Y. Generic assignments, strain histories and properties of pure cultures of cyanobacteria. J. Gen. Microbiol. 111, 1-61 (1979).

81. Shigi, N., Suzuki, T., Tamakoshi, M., Oshima, T. \& Watanabe, K. Conserved bases in the TPsi C loop of tRNA are determinants for thermophile-specific 2thiouridylation at position 54. J. Biol. Chem. 277, 39128-39135 (2002).

82. Kang, B. I. et al. Identification of 2-methylthio cyclic N6threonylcarbamoyladenosine (ms2ct6A) as a novel RNA modification at position 37 of tRNAs. Nucleic Acids Res. 45, 2124-2136 (2017).

83. Taniguchi, T. et al. Decoding system for the AUA codon by tRNAIle with the UAU anticodon in Mycoplasma mobile. Nucleic Acids Res. 41, 2621-2631 (2013).

84. Pyzocha, N. K., Ran, F. A., Hsu, P. D. \& Zhang, F. RNA-guided genome editing of mammalian cells. Methods Mol. Biol. 1114, 269-277 (2014).

85. Ran, F. A. et al. Genome engineering using the CRISPR-Cas9 system. Nat. Protoc. 8, 2281-2308 (2013).

86. Ishiguro, K., Arai, T. \& Suzuki, T. Depletion of S-adenosylmethionine impacts on ribosome biogenesis through hypomodification of a single rRNA methylation. Nucleic Acids Res. 47, 4226-4239 (2019).

87. Miyauchi, K., Ohara, T. \& Suzuki, T. Automated parallel isolation of multiple species of non-coding RNAs by the reciprocal circulating chromatography method. Nucleic Acids Res. 35, e24 (2007).

88. Sakaguchi, Y., Miyauchi, K., Kang, B. I. \& Suzuki, T. Nucleoside analysis by hydrophilic interaction liquid chromatography coupled with mass spectrometry. Methods Enzymol. 560, 19-28 (2015).

89. Milligan, J. F., Groebe, D. R., Witherell, G. W. \& Uhlenbeck, O. C. Oligoribonucleotide synthesis using T7 RNA polymerase and synthetic DNA templates. Nucleic Acids Res. 15, 8783-8798 (1987).

90. Malapaka, R. R., Adebayo, L. O. \& Tripp, B. C. A deletion variant study of the functional role of the Salmonella flagellin hypervariable domain region in motility. J. Mol. Biol. 365, 1102-1116 (2007).

91. Foster, P. L. Methods for determining spontaneous mutation rates. Methods Enzymol. 409, 195-213 (2006).

92. Ishizawa, Y., Ying, B. W., Tsuru, S. \& Yomo, T. Nutrient-dependent growth defects and mutability of mutators in Escherichia coli. Genes Cells 20,68-76 (2015).

93. McLuckey, S. A., Van Berkel, G. J. \& Glish, G. L. Tandem mass spectrometry of small, multiply charged oligonucleotides. J. Am. Soc. Mass Spectrom. 3, 60-70 (1992)

\section{Acknowledgements}

We are grateful to the current and former members of the Suzuki laboratory, especially to Yuriko Sakaguchi, Satoshi Kimura, and Hiroki Kawai, for many fruitful discussions and technical advices. This work was supported by Grants-in-Aid for Scientific Research on Priority Areas from the Ministry of Education, Science, Sports, and Culture of Japan (T.S. and K.M.).

\section{Author contributions}

M.T. and K.I. mainly performed most of experiments. S.A. and K.M. assisted genetic and biochemical works. All authors discussed the results. K.M. and T.S. designed the research. K.I., M.T. and T.S. wrote this paper. T.S. supervised all the work.

\section{Competing interests}

The authors declare no competing interests.

\section{Additional information}

Supplementary information is available for this paper at https://doi.org/10.1038/s41467019-13525-3.

Correspondence and requests for materials should be addressed to T.S.

Peer review information Nature Communications thanks the anonymous reviewers for their contribution to the peer review of this work. Peer reviewer reports are available.

Reprints and permission information is available at http://www.nature.com/reprints

Publisher's note Springer Nature remains neutral with regard to jurisdictional claims in published maps and institutional affiliations.

Open Access This article is licensed under a Creative Commons Attribution 4.0 International License, which permits use, sharing, adaptation, distribution and reproduction in any medium or format, as long as you give appropriate credit to the original author(s) and the source, provide a link to the Creative Commons license, and indicate if changes were made. The images or other third party material in this article are included in the article's Creative Commons license, unless indicated otherwise in a credit line to the material. If material is not included in the article's Creative Commons license and your intended use is not permitted by statutory regulation or exceeds the permitted use, you will need to obtain permission directly from the copyright holder. To view a copy of this license, visit http://creativecommons.org/ licenses/by/4.0/.

(c) The Author(s) 2019 